\title{
Decoding (pseudo)-scalar operators in leptonic and semileptonic $B$ decays
}

\author{
Giovanni Banelli ${ }^{1}$, Robert Fleischer ${ }^{1,2}$, Ruben Jaarsma ${ }^{1, a}$, Gilberto Tetlalmatzi-Xolocotzi ${ }^{1}$ \\ ${ }^{1}$ Nikhef, Science Park 105, 1098 XG Amsterdam, The Netherlands \\ ${ }^{2}$ Faculty of Science, Vrije Universiteit Amsterdam, 1081 HV Amsterdam, The Netherlands
}

Received: 2 October 2018 / Accepted: 27 October 2018 / Published online: 10 November 2018

(C) The Author(s) 2018

\begin{abstract}
We consider leptonic $B^{-} \rightarrow \ell^{-} \bar{v}_{\ell}$ and semileptonic $\bar{B} \rightarrow \pi \ell^{-} \bar{\nu}_{\ell}, \bar{B} \rightarrow \rho \ell^{-} \bar{\nu}_{\ell}$ decays and present a strategy to determine short-distance coefficients of New-Physics operators and the CKM element $\left|V_{u b}\right|$. As the leptonic channels play a central role, we illustrate this method for (pseudo)scalar operators which may lift the helicity suppression of the corresponding transition amplitudes arising in the Standard Model. Utilising a new result by the Belle collaboration for the branching ratio of $B^{-} \rightarrow \mu^{-} \bar{v}_{\mu}$, we explore theoretically clean constraints and correlations between New Physics coefficients for leptonic final states with $\mu$ and $\tau$ leptons. In order to obtain stronger bounds and to extract $\left|V_{u b}\right|$, we employ semileptonic $\bar{B} \rightarrow \pi \ell^{-} \bar{\nu}_{\ell}$ and $\bar{B} \rightarrow \rho \ell^{-} \bar{\nu}_{\ell}$ decays as an additional ingredient, involving hadronic form factors which are determined through QCD sum rule and lattice calculations. In addition to a detailed analysis of the constraints on the New Physics contributions following from current data, we make predictions for yet unmeasured decay observables, compare them with experimental constraints and discuss the impact of CP-violating phases of the New-Physics coefficients.
\end{abstract}

\section{Introduction}

Leptonic transitions of $B$ mesons are the simplest weak decay class as the final-state particles do not have $S U(3)_{\mathrm{C}}$ colour quantum numbers. Consequently, the whole hadron dynamics is described by a single parameter, the $B$-meson decay constant

$\left\langle 0\left|\bar{u} \gamma_{\mu} \gamma_{5} b\right| B^{-}(p)\right\rangle=i f_{B^{-}} p_{\mu}$,

which can be determined through lattice-QCD methods. While leptonic decays of neutral $B$ mesons are rare processes originating from flavour-changing neutral currents, those of

a e-mail: rjaarsma@nikhef.nl charged mesons, $B^{-} \rightarrow \ell^{-} \bar{v}_{\ell}$, are caused by charged-current interactions (with $\ell=e, \mu, \tau$ ). Within the Standard Model $(\mathrm{SM})$, the branching ratio takes the following form:

$\left.\mathcal{B}\left(B^{-} \rightarrow \ell^{-} \bar{v}_{\ell}\right)\right|_{\mathrm{SM}}=\frac{G_{\mathrm{F}}^{2}}{8 \pi}\left|V_{u b}\right|^{2} M_{B^{-}} m_{\ell}^{2}\left(1-\frac{m_{\ell}^{2}}{M_{B^{-}}^{2}}\right)^{2} f_{B^{-}}^{2} \tau_{B^{-}}$,

where $G_{\mathrm{F}}$ is Fermi's constant, $\tau_{B^{-}}$the lifetime of the $B^{-}$ meson, $M_{B^{-}}$and $m_{\ell}$ are the $B^{-}$and lepton masses, respectively, and the neutrino mass has been neglected. This branching ratio is suppressed by the Cabibbo-Kobayashi-Maskawa (CKM) element $V_{u b}$, and exhibits a helicity suppression, which is reflected by the proportionality to $m_{\ell}^{2}$. Using $f_{B^{-}}=$ $0.186 \pm 0.004 \mathrm{GeV}$ [1] and assuming the SM with [2]

$\left|V_{u b}\right|=(3.601 \pm 0.098) \times 10^{-3}$,

we obtain

$$
\begin{aligned}
\left.\mathcal{B}\left(B^{-} \rightarrow \tau^{-} \bar{v}_{\tau}\right)\right|_{\mathrm{SM}} & =(7.92 \pm 0.55) \times 10^{-5} \\
\left.\mathcal{B}\left(B^{-} \rightarrow \mu^{-} \bar{v}_{\mu}\right)\right|_{\mathrm{SM}} & =(3.56 \pm 0.25) \times 10^{-7} \\
\left.\mathcal{B}\left(B^{-} \rightarrow e^{-} \bar{v}_{e}\right)\right|_{\mathrm{SM}} & =(8.33 \pm 0.58) \times 10^{-12}
\end{aligned}
$$

In the case of $\ell=\tau$, the helicity suppression is very ineffective due to the large $\tau$ mass. Consequently, despite the challenging $\tau$ reconstruction, the $B^{-} \rightarrow \tau^{-} \bar{\nu}_{\tau}$ mode could already be observed by the BaBar and Belle collaborations about a decade ago, with the current average by the Particle Data Group (PDG) given as follows [3]:

$\mathcal{B}\left(B^{-} \rightarrow \tau^{-} \bar{\nu}_{\tau}\right)=(1.09 \pm 0.24) \times 10^{-4}$.

On the other hand, $B^{-} \rightarrow \mu^{-} \bar{v}_{\mu}$ and $B^{-} \rightarrow e^{-} \bar{v}_{e}$ with their SM branching ratios in regimes of $10^{-7}$ and $10^{-11}$, respectively, appear much more challenging to measure. Nevertheless, the Belle collaboration has recently performed a new search for the former channel, finding a $2.4 \sigma$ excess over the background [4]: 


$$
\begin{aligned}
\mathcal{B} & \left(B^{-} \rightarrow \mu^{-} \bar{v}_{\mu}\right) \\
& =\left(6.46 \pm\left. 2.22\right|_{\text {stat }} \pm 1.60_{\text {syst }}\right) \times 10^{-7} \\
& =(6.46 \pm 2.74) \times 10^{-7}
\end{aligned}
$$

For the electronic channel, only an upper bound is available, which was obtained by the Belle collaboration in 2007 [5]:

$\mathcal{B}\left(B^{-} \rightarrow e^{-} \bar{v}_{e}\right)<9.8 \times 10^{-7}(90 \%$ C.L. $)$.

Decays of $B$ mesons into final states with $\tau$ leptons are receiving a lot of interest in view of experimental results which indicate possible signals of New Physics (NP), where the ratios $R_{D^{(*)}}$ of the branching ratios of $\bar{B} \rightarrow D^{(*)} \tau^{-} \bar{v}_{\tau}$ and $\bar{B} \rightarrow D^{(*)} \mu^{-} \bar{v}_{\mu}$ decays are in the focus (see, for instance, Refs. [6-13] and references therein). The exciting feature is an indication of a violation of the universality between $\tau$ and $\mu$. These results are complemented by measurements of the ratios of branching ratios of the rare decays $B \rightarrow K^{(*)} \mu^{+} \mu^{-}$ and $B \rightarrow K^{(*)} e^{+} e^{-}$which may signal a violation of the universality of muons and electrons in these processes (for recent overviews, see Refs. [14, 15]).

In this paper, we propose a new strategy to probe NP effects by utilising leptonic $B$ decays and the interplay with their semileptonic counterparts. Both decay classes are actually caused by the same low-energy effective Hamiltonian. We will obtain constraints on short-distance coefficients using the Belle result in Eq. (8) and address the question of how large the branching ratio of $B^{-} \rightarrow e^{-} \bar{v}_{e}$ could be due to a lift of the helicity suppression through NP effects. We have addressed a similar question for the leptonic rare decays $B_{s, d}^{0} \rightarrow e^{+} e^{-}$, which could be enhanced to the level of the $B_{s, d}^{0} \rightarrow \mu^{+} \mu^{-}$channels through new (pseudo)-scalar interactions [16]. We shall also make predictions for various semileptonic decay ratios which will allow us to fully reveal the underlying decay dynamics, and extract $\left|V_{u b}\right|$ while also allowing for NP contributions.

A subtle point is given by $\mathrm{CP}$-violating phases which may be present in the short-distance coefficients of NP operators. As is well known from discussions of non-leptonic meson decays, CP-violating asymmetries arising directly at the decay amplitude level,

$a_{\mathrm{CP}} \equiv \frac{\mathcal{B}(\bar{B} \rightarrow \bar{f})-\mathcal{B}(B \rightarrow f)}{\mathcal{B}(\bar{B} \rightarrow \bar{f})+\mathcal{B}(B \rightarrow f)}$,

are induced by the interference between decay amplitudes with both non-trivial $\mathrm{CP}$-violating and non-trivial $\mathrm{CP}$ conserving phase differences [17]. While the former originate from phases of CKM matrix elements in the SM or possible CP-violating NP phases, the latter could be generated through strong interactions or absorptive parts of loop diagrams. In the SM, the direct $\mathrm{CP}$ asymmetries vanish hence in (semi)-leptonic decays at leading order in the weak interactions while higher-order-effects can only gener- ate negligible effects [18-21]. Due to the lack of sizeable CPconserving phase differences, direct CP asymmetries (10) of $B^{-} \rightarrow \ell^{-} \bar{v}_{\ell}, \bar{B} \rightarrow \pi \ell^{-} \bar{v}_{\ell}$, and $\bar{B} \rightarrow \rho \ell^{-} \bar{\nu}_{\ell}$ decays can also not take sizeable values in the presence of NP contributions. Consequently, we cannot get empirical evidence for such phases through possible direct CP asymmetries in such modes, in contrast to non-leptonic $B$ decays where strong interactions are at work to generate strong phase differences. On the other hand, in the leptonic rare $\bar{B}_{q}^{0} \rightarrow \ell^{+} \ell^{-}$decays of neutral $\bar{B}_{q}^{0}$ mesons $(q=d, s)$, the impact of $B_{q}^{0}-\bar{B}_{q}^{0}$ mixing may induce $\mathrm{CP}$-violating asymmetries, thereby indicating possible CP-violating NP phases [22].

Contributions of NP to $B^{-} \rightarrow \ell^{-} \bar{v}_{\ell}, \bar{B} \rightarrow \pi \ell^{-} \bar{v}_{\ell}$ and $\bar{B} \rightarrow \rho \ell^{-} \bar{v}_{\ell}$ processes have also been addressed in, e.g., Refs. [23-28].

The outline of this paper is as follows: after introducing briefly the theoretical framework in Sect. 2, we discuss the leptonic $B^{-} \rightarrow \ell^{-} \bar{v}_{\ell}$ decays in Sect. 3. The semileptonic $\bar{B} \rightarrow \rho \ell^{-} \bar{v}_{\ell}$ and $\bar{B} \rightarrow \pi \ell^{-} \bar{v}_{\ell}$ modes are analysed in Sect. 4, where we will also combine them with the leptonic constraints to obtain regions for short-distance coefficients. The hadronic form factors, which are required for the study of experimental data, are discussed in Appendix A. In both Sects. 3 and 4 we will also address the impact of $\mathrm{CP}$ violating phases on the regions for the short-distance coefficients. Then, in Sect. 5 we determine $\left|V_{u b}\right|$ in the presence of NP contributions. Finally, we give predictions for the not yet measured branching ratios $\mathcal{B}\left(B^{-} \rightarrow \mu^{-} \bar{v}_{\mu}\right)$, $\mathcal{B}\left(\bar{B} \rightarrow \rho \tau^{-} \bar{v}_{\tau}\right)$, and $\mathcal{B}\left(\bar{B} \rightarrow \pi \tau^{-} \bar{v}_{\tau}\right)$ in Sect. 6. The conclusions are summarised in Sect. 7 .

\section{Theoretical framework}

In the SM, the leptonic decays

$B^{-} \rightarrow \ell^{-} \bar{v}_{\ell}$,

(with $\ell=e, \mu, \tau$ ) originate from charged-current interactions due to the $W^{-}$exchange between quark and lepton currents, which are effectively described by the four-fermion operator

$\mathcal{O}_{V_{L}}^{\ell}=\left(\bar{q} \gamma^{\mu} P_{L} b\right)\left(\bar{\ell} \gamma_{\mu} P_{L} v_{\ell}\right)$

The $\mathcal{O}_{V_{L}}^{\ell}$ operator also contributes to semileptonic transitions. For instance, for $q=u$, we have

$$
\begin{array}{ll}
B^{-} \rightarrow \rho^{0} \ell^{-} \bar{v}_{\ell}, & \bar{B}^{0} \rightarrow \rho^{+} \ell^{-} \bar{v}_{\ell}, \\
B^{-} \rightarrow \pi^{0} \ell^{-} \bar{v}_{\ell}, & \bar{B}^{0} \rightarrow \pi^{+} \ell^{-} \bar{v}_{\ell} .
\end{array}
$$

In extensions of the SM, interactions with NP particles may lead to 


$$
\begin{gathered}
\mathcal{O}_{S}^{\ell}=(\bar{q} b)\left(\bar{\ell} P_{L} v_{\ell}\right), \quad \mathcal{O}_{P}^{\ell}=\left(\bar{q} \gamma_{5} b\right)\left(\bar{\ell} P_{L} v_{\ell}\right), \\
\mathcal{O}_{V_{R}}^{\ell}=\left(\bar{q} \gamma^{\mu} P_{R} b\right)\left(\bar{\ell} \gamma_{\mu} P_{L} v_{\ell}\right), \\
\mathcal{O}_{T}^{\ell}=\left(\bar{q} \sigma^{\mu \nu} P_{L} b\right)\left(\bar{\ell} \sigma_{\mu \nu} P_{L} v_{\ell}\right),
\end{gathered}
$$

where $\mathcal{O}_{S}^{\ell}, \mathcal{O}_{P}^{\ell}, \mathcal{O}_{V_{R}}^{\ell}$ and $\mathcal{O}_{T}^{\ell}$ correspond to a scalar, pseudoscalar, (an extra) vector, and a tensor operator, respectively. Notice that we are assuming the neutrinos to be left-handed and to have the same flavour as the lepton in each one of the operators.

We utilize the recent result by the Belle collaboration for $\mathcal{B}\left(B^{-} \rightarrow \mu^{-} \bar{v}_{\mu}\right)$ [4] given in Eq. (8). Combining this observable with experimental data for $B^{-} \rightarrow \tau^{-} \bar{\nu}_{\tau}$ and the semileptonic channels in Eq. (13), we are in a position to probe lepton flavour universality in decays mediated by a $b \rightarrow u \ell \bar{v}_{\ell}$ transition. This is complimentary to the $R_{D^{(*)}}$ observables, which involve $b \rightarrow c \ell \bar{v}_{\ell}$ transitions. New vector currents are often considered to explain the experimental measurements of these observables. Here we concentrate on the study of the effects of $\mathcal{O}_{S}^{\ell}$ and $\mathcal{O}_{P}^{\ell}$. Due to the structure of the formulae, they lift the helicity suppression of the leptonic decays. Consequently, these channels put strong constraints on the corresponding short-distance coefficients, while still allowing for interesting phenomenological predictions. We then obtain the following low-energy effective Hamiltonian:

$\mathcal{H}_{\text {eff }}=\frac{4 G_{\mathrm{F}}}{\sqrt{2}} V_{q b}\left[C_{V_{L}} \mathcal{O}_{V_{L}}^{\ell}+C_{S}^{\ell} \mathcal{O}_{S}^{\ell}+C_{P}^{\ell} \mathcal{O}_{P}^{\ell}\right]+$ h.c.

In our analysis the vector coefficient takes its $\mathrm{SM}$ value $C_{V_{L}}=1$.

A prominent example of such NP contributions is the effect of charged Higgs bosons which arise in the context of type II Two-Higgs-Doublet-Models (2HDM) [29], where

$C_{P}^{\ell}=C_{S}^{\ell}=-\tan ^{2} \beta\left(\frac{m_{b} m_{\ell}}{M_{H^{ \pm}}^{2}}\right)$.

Here, $\tan \beta$ is the ratio of the vacuum expectation values and $M_{H^{ \pm}}$denotes the mass of the charged Higgs boson. A more recent discussion using the Georgi-Machacek model was given in Ref. [30], together with scenarios having leptoquarks.

\section{Leptonic $B^{-} \rightarrow \ell^{-} \bar{v}_{\ell}$ decays}

Using the Hamiltonian in Eq. (15) we obtain the following branching ratio for the leptonic decays [31]:

$$
\begin{aligned}
& \mathcal{B}\left(B^{-} \rightarrow \ell^{-} \bar{v}_{\ell}\right) \\
& =\left.\mathcal{B}\left(B^{-} \rightarrow \ell^{-} \bar{v}_{\ell}\right)\right|_{\mathrm{SM}}\left|1+\frac{M_{B^{-}}^{2}}{m_{\ell}\left(m_{b}+m_{u}\right)} C_{P}^{\ell}\right|^{2},
\end{aligned}
$$

where the prefactor $\left.\mathcal{B}\left(B^{-} \rightarrow \ell^{-} \bar{v}_{\ell}\right)\right|_{\mathrm{SM}}$ is the SM branching ratio, which is given in Eq. (2). Here $m_{b}$ and $m_{u}$ are quark masses which enter through the use of the equations of motion of the quark fields. To get a better understanding of the effect of the NP contributions to Eq. (17), we rewrite this expression as

$$
\begin{aligned}
& \mathcal{B}\left(B^{-} \rightarrow \ell^{-} \bar{\nu}_{\ell}\right)=\frac{G_{\mathrm{F}}^{2}}{8 \pi}\left|V_{u b}\right|^{2} M_{B^{-}}^{3} f_{B^{-}}^{2} \tau_{B^{-}}\left(1-\frac{m_{\ell}^{2}}{M_{B^{-}}^{2}}\right)^{2} \\
& \quad \times\left[\left(\frac{m_{\ell}}{M_{B^{-}}}\right)^{2}+2 \frac{m_{\ell}}{M_{B^{-}}}\left(\frac{M_{B^{-}}}{m_{b}+m_{u}}\right) \Re\left(C_{P}^{\ell}\right)\right. \\
& \left.\quad+\left(\frac{M_{B^{-}}}{m_{b}+m_{u}}\right)^{2}\left|C_{P}^{\ell}\right|^{2}\right],
\end{aligned}
$$

where we can see how the term proportional to $\left|C_{P}^{\ell}\right|^{2}$ may potentially play a dominant role since it is not suppressed by powers of $m_{\ell} / M_{B^{-}}$.

There is a subtlety related with Eqs. (17) and (18) when allowing for physics beyond the SM: the point is that the value of $\left|V_{u b}\right|$ extracted from sophisticated analyses of semileptonic $B$ decays (for an overview, see Ref. [3]) may include NP contributions, thereby precluding us from calculating the SM branching ratio. In order to deal with this issue, our analysis will be based on the study of ratios of branching fractions.

\subsection{Constraints on pseudoscalar NP coefficients from leptonic decay observables}

We start our analysis by determining bounds for the pseudoscalar Wilson coefficient $C_{P}^{\ell}$. To this end, we consider the ratio of two leptonic decays to obtain

$$
\begin{aligned}
R_{\ell_{2}}^{\ell_{1}} & \equiv \frac{m_{\ell_{2}}^{2}}{m_{\ell_{1}}^{2}}\left(\frac{M_{B^{-}}^{2}-m_{\ell_{2}}^{2}}{M_{B^{-}}^{2}-m_{\ell_{1}}^{2}}\right)^{2} \frac{\mathcal{B}\left(B^{-} \rightarrow \ell_{1}^{-} \bar{v}_{\ell_{1}}\right)}{\mathcal{B}\left(B^{-} \rightarrow \ell_{2}^{-} \bar{v}_{\ell_{2}}\right)} \\
& =\left|\frac{1+\mathcal{C}_{\ell_{1} ; P}}{1+\mathcal{C}_{\ell_{2} ; P}}\right|^{2}
\end{aligned}
$$

with

$\mathcal{C}_{\ell ; P} \equiv\left|\mathcal{C}_{\ell ; P}\right| e^{i \phi_{\ell}}=\left[\frac{M_{B^{-}}^{2}}{m_{\ell}\left(m_{b}+m_{q}\right)}\right] C_{P}^{\ell}$,

where we have allowed for a generic CP-violating phase $\phi_{\ell}^{q}$. We would like to highlight some interesting features of $R_{\ell_{\ell}}^{\ell_{1}}$ : unlike the leptonic branching ratios themselves, this quantity has the advantage that it does not depend on $\left|V_{u b}\right|$. Moreover, it is theoretically clean as the decay constants cancel. Finally, in the absence of NP contributions, corresponding to $C_{P}^{\ell_{1}}=$ $C_{P}^{\ell_{2}}=0$, we have by definition $\left.R_{\ell_{2}}^{\ell_{1}}\right|_{\mathrm{SM}}=1$.

Let us first assume that the NP short-distance coefficients $C_{P}^{\ell}$ in Eq. (20) are real, and study the constraints we obtain from the leptonic decay ratios defined in Eq. (19). For the specific determination of $R_{\ell_{2}}^{\ell_{1}}$, we consider the tau-muon and the electron-muon pairs, i.e. $R_{\mu}^{\tau}$ and $R_{\mu}^{e}$, respectively. 


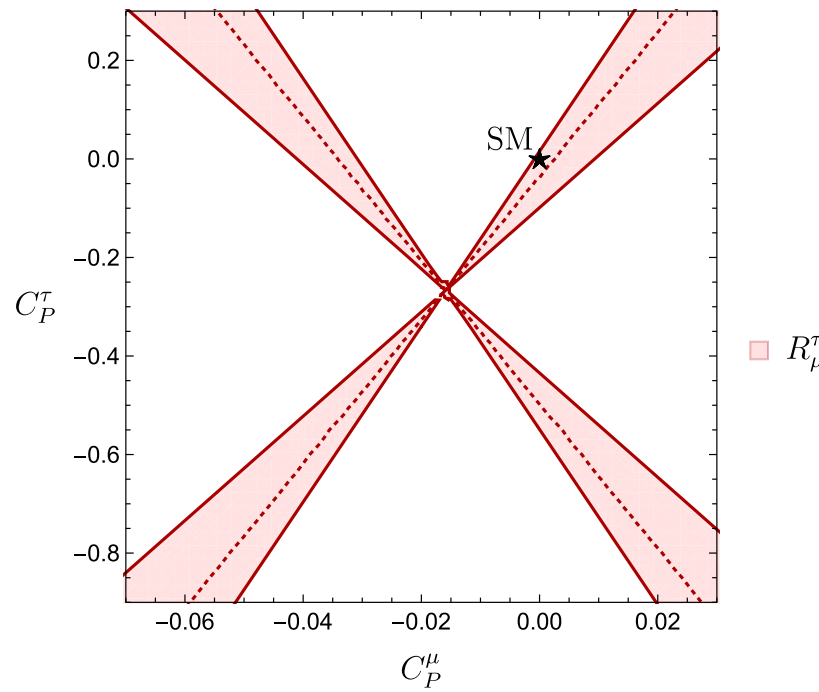

Fig. 1 Allowed regions in the $C_{P}^{\mu}-C_{P}^{\tau}$ plane following from the leptonic ratio $R_{\mu}^{\tau}$

We obtain the experimental value of $R_{\mu}^{\tau}$ using as numerical inputs Eqs. (7) and (8), yielding

$R_{\mu}^{\tau}=0.76 \pm 0.36$.

By comparing this experimental result with the corresponding theoretical expression, we determine the allowed regions in the $C_{P}^{\mu}-C_{P}^{\tau}$ plane, resulting in the cross-shaped area shown in Fig. 1. Here and throughout the rest of this work, the dotted lines define the central value of the corresponding observable, and the $1 \sigma$ allowed regions are bounded by the solid lines. It is interesting to note that the constraints obtained are in agreement with the SM point $\left(C_{P}^{\mu}=C_{P}^{\tau}=0\right)$, which is indicated by the black star in Fig. 1.

To calculate $R_{\mu}^{e}$, we require $\mathcal{B}\left(B^{-} \rightarrow e^{-} \bar{v}_{e}\right)$ and $\mathcal{B}\left(B^{-} \rightarrow \mu^{-} \bar{v}_{\mu}\right)$. For the former only the upper bound in Eq. (9) is available. Since this quantity defines the numerator in $R_{\mu}^{e}$, we obtain the experimental bound

$R_{\mu}^{e}<6.48 \times 10^{4}$,

with an overall error of $\pm 2.75 \times 10^{4}$ induced by the uncertainty associated with $\mathcal{B}\left(B^{-} \rightarrow \mu^{-} \bar{v}_{\mu}\right)$. Let us now determine the allowed regions in the $C_{P}^{\mu}-C_{P}^{e}$ plane derived from the experimental bound in Eq. (22). The result is given by the wedge-shaped regions in Fig. 2, which contain the SM point $C_{P}^{\mu}=C_{P}^{e}=0$. A future measurement of $\mathcal{B}\left(B^{-} \rightarrow e^{-} \bar{v}_{e}\right)$ will allow us to determine stringent constraints from $R_{\mu}^{e}$. In that case, we expect a cross-shaped region analogous to the one found for $R_{\mu}^{\tau}$ in Fig. 1 .

As discussed in Sect. 2, an important NP scenario that leads to new pseudo-scalar effects in semileptonic decays is the 2HDM. It is instructive to have a closer look at the impact of this scenario on the ratio $R_{\ell_{2}}^{\ell_{1}}$ defined in Eq. (19), yielding

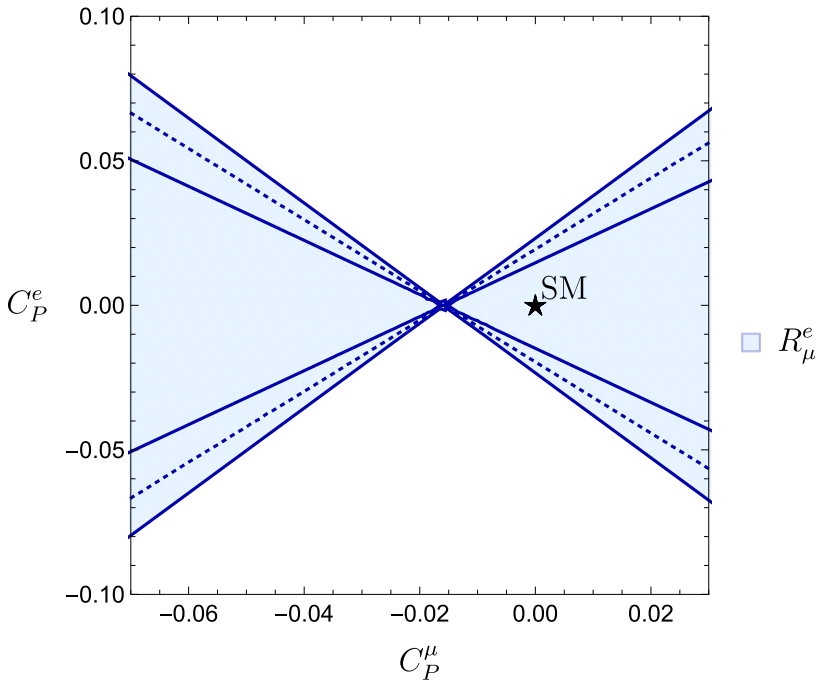

Fig. 2 Allowed regions in the $C_{P}^{\mu}-C_{P}^{e}$ plane following from the leptonic ratio $R_{\mu}^{e}$. The dotted line defines where the bound in Eq. (22) is saturated, with an error indicated by the solid lines due to the uncertainty of the measurement of $\mathcal{B}\left(B^{-} \rightarrow \mu^{-} \bar{v}_{\mu}\right)$

$\left.\mathcal{C}_{\ell ; P}\right|_{2 \mathrm{HDM}}=-\tan ^{2} \beta\left(\frac{M_{B^{-}}}{M_{H^{ \pm}}}\right)^{2} \frac{m_{b}}{m_{b}+m_{u}}$.

The right-hand side does actually not depend on the lepton flavour $\ell$, i.e.

$\left.\mathcal{C}_{\mu ; P}\right|_{2 \mathrm{HDM}}=\left.\mathcal{C}_{e ; P}\right|_{2 \mathrm{HDM}}=\left.\mathcal{C}_{\tau ; P}\right|_{2 \mathrm{HDM}}$,

leading to the pattern

$\left.R_{\mu}^{\tau}\right|_{2 \mathrm{HDM}}=\left.R_{\mu}^{e}\right|_{2 \mathrm{HDM}}=1$,

as in the SM.

\subsection{Implications of CP-violating phases}

Let us now explore the impact of CP-violating phases of the NP coefficients $C_{P}^{\ell}$. To this end, we write

$C_{P}^{\ell}=\left|C_{P}^{\ell}\right| e^{i \phi_{P}^{\ell}}$,

where $\phi_{P}^{\ell}$ coincides with the CP-violating phase of $\mathcal{C}_{\ell ; P}^{u}$ in Eq. (20), and obtain

$R_{\ell_{2}}^{\ell_{1}}=\frac{1+2\left|\mathcal{C}_{\ell_{1} ; P}\right| \cos \phi_{\ell_{1} ; P}+\left|\mathcal{C}_{\ell_{1} ; P}\right|^{2}}{1+2\left|\mathcal{C}_{\ell_{2} ; P}\right| \cos \phi_{\ell_{2}}+\left|\mathcal{C}_{\ell_{2} ; P}\right|^{2}}$.

We may now convert the experimental value for $R_{\ell_{2}}^{\ell_{1}}$ into a correlation between $\left|\mathcal{C}_{\ell_{1} ; P}\right|$ and $\left|\mathcal{C}_{\ell_{2} ; P}\right|$ for given combinations of the $\mathrm{CP}$-violating phases $\phi_{\ell_{1}}^{u}$ and $\phi_{\ell_{2}}^{u}$ :

$$
\begin{aligned}
& \left|\mathcal{C}_{\ell_{1} ; P}\right| \\
& =-\cos \phi_{\ell_{1}} \pm \sqrt{R_{\ell_{2}}^{\ell_{1}}\left[1+2\left|\mathcal{C}_{\ell_{2} ; P}\right| \cos \phi_{\ell_{2}}+\left|\mathcal{C}_{\ell_{2}}\right|^{2}\right]-\sin ^{2} \phi_{\ell_{1}}} .
\end{aligned}
$$


Assuming real coefficients, i.e. $\phi_{\mu}^{u}, \phi_{\tau}^{u} \in\left\{0^{\circ}, 180^{\circ}\right\}$, yields

$\left|\mathcal{C}_{\ell_{1} ; P}\right|=\mp 1 \pm \sqrt{R_{\ell_{2}}^{\ell_{1}}}|1 \pm| \mathcal{C}_{\ell_{2} ; P}||$,

which results in a linear correlation between $\left|\mathcal{C}_{\ell_{1} ; P}\right|$ and $\left|\mathcal{C}_{\ell_{2} ; P}\right|$.

Mapping Eq. (27) to the observable $R_{\mu}^{\tau}$ leads to four unknown parameters: $\left|C_{P}^{\mu}\right|, \phi_{P}^{\mu},\left|C_{P}^{\tau}\right|$ and $\phi_{P}^{\tau}$. Therefore, in order to study the correlation between $\left|C_{P}^{\mu}\right|$ and its complex phase $\phi_{P}^{\mu}$, we have to make an assumption for $\left|C_{P}^{\tau}\right|$ and $\phi_{P}^{\tau}$. In the case of universal Wilson coefficients for muons and taus, satisfying the relation

$C_{P}^{\mu}=C_{P}^{\tau}$,

we find

$\left(\frac{M_{B^{-}}}{m_{b}+m_{u}}\right)\left|C_{P}^{\mu}\right|=-a \pm \sqrt{a^{2}-b}$

with

$$
\begin{aligned}
a & \equiv\left[\frac{\frac{M_{B^{-}}}{m_{\tau}}-R_{\mu}^{\tau} \frac{M_{B^{-}}}{m_{\mu}}}{\left(\frac{M_{B^{-}}}{m_{\tau}}\right)^{2}-R_{\mu}^{\tau}\left(\frac{M_{B^{-}}}{m_{\mu}}\right)^{2}}\right] \cos \phi_{P}^{\mu}, \\
b & =\frac{1-R_{\mu}^{\tau}}{\left(\frac{M_{B^{-}}}{m_{\tau}}\right)^{2}-R_{\mu}^{\tau}\left(\frac{M_{B^{-}}}{m_{\mu}}\right)^{2}} .
\end{aligned}
$$

The resulting correlation is shown in Fig. 3.

On the other hand, assuming

$C_{P}^{\mu}=C_{P}^{e}$,

yields the constraint from $R_{\mu}^{e}$ shown in Fig. 4. By looking at Figs. 3 and 4 , we can see how the SM point $\left|C_{P}^{\mu}\right|=\phi_{P}^{\mu}=0$ is consistent with the constraints derived from the current data.

It is also interesting to explore the correlations in the $\left|C_{P}^{\mu}\right|-$ $\left|C_{P}^{\tau}\right|$ plane that arise for different combinations of the CPviolating phases $\phi_{P}^{\mu}$ and $\phi_{P}^{\tau}$. For example, assuming $\phi_{P}^{\tau}=0^{\circ}$ and considering different values of $\phi_{P}^{\mu}$, we obtain the patterns shown in the left panel of Fig. 5. On the other hand, the right panel shows how the contours are affected when we keep $\phi_{P}^{\mu}=0^{\circ}$ and vary $\phi_{P}^{\tau}$. For $\phi_{P}^{\mu, \tau}=0^{\circ}, 180^{\circ}$ the central values of the observables in Fig. 5 obey the linear correlation indicated in Eq. (29).

\section{Semileptonic $\bar{B} \rightarrow \rho \ell^{-} \bar{\nu}_{\ell}$ and $\bar{B} \rightarrow \pi \ell^{-} \bar{v}_{\ell}$ decays}

We may improve the constraints on the NP short-distance contributions if in addition to the leptonic processes described in Sect. 3 we also include semileptonic decays caused by the transition $b \rightarrow u \ell^{-} \bar{v}_{\ell}$. The relevant decays for our analysis are $\bar{B} \rightarrow \rho \ell^{-} \bar{v}_{\ell}$ and $\bar{B} \rightarrow \pi \ell^{-} \bar{\nu}_{\ell}$. The first mode depends

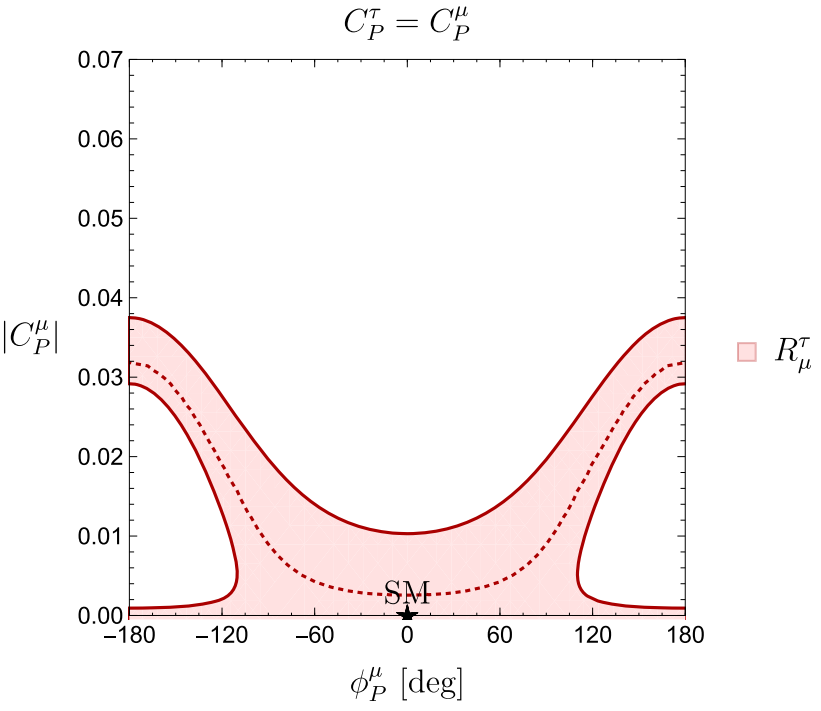

Fig. 3 New physics regions in the $\phi_{P}^{\mu}-\left|C_{P}^{\mu}\right|$ plane, assuming flavour universality for the pseudoscalar Wilson coefficients of $\mu$ and $\tau$

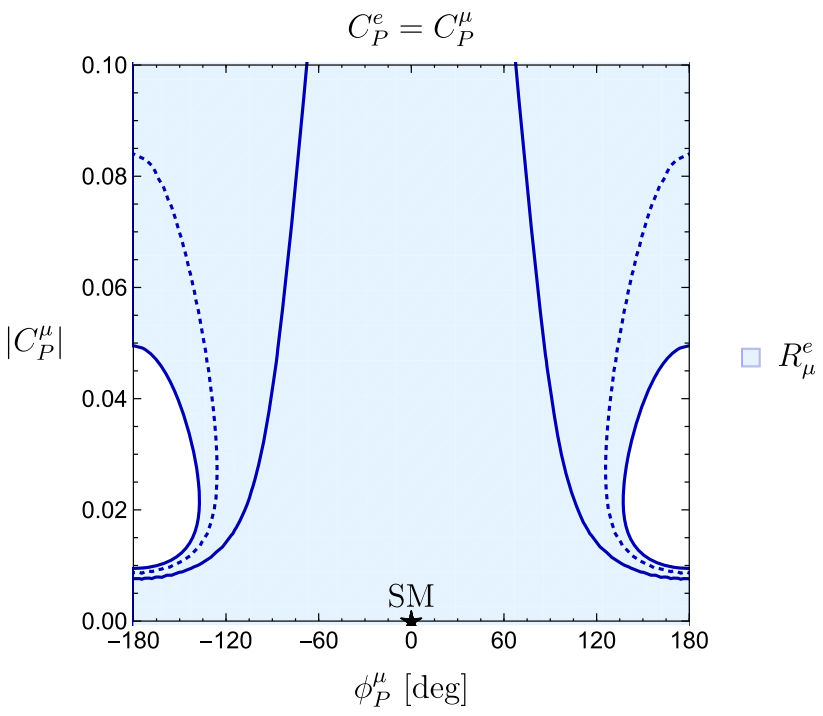

Fig. 4 New physics regions in the $\phi_{P}^{\mu}-\left|C_{P}^{\mu}\right|$ plane, assuming flavour universality for the pseudoscalar Wilson coefficients of $\mu$ and $e$

only on $C_{P}^{\ell}$ and therefore can be considered the counterpart of the leptonic channels. On the other hand, the process $\bar{B} \rightarrow \pi \ell^{-} \bar{\nu}_{\ell}$ is sensitive to the short distance contribution $C_{S}^{\ell}$.

The expressions for semileptonic decays have a more complicated structure than those for the leptonic modes due to the hadronic form factors used to calculate the transitions $\bar{B} \rightarrow \rho$ and $\bar{B} \rightarrow \pi$. The kinematical regimes for the semileptonic decays are described in terms of $q^{2} \equiv\left(p_{B}-p_{\rho, \pi}\right)^{2}$, where $p_{B}$ and $p_{\rho, \pi}$ are the four-momenta of the $B$-meson and the $\rho$ or $\pi$, respectively. For low momentum transfer, i.e. $q^{2} \in\left[0, q_{\max }^{2}\right]$ where $12 \mathrm{GeV}^{2} \leq q_{\max }^{2} \leq 16 \mathrm{GeV}^{2}$, the nonperturbative hadronic form factors are estimated using QCD 

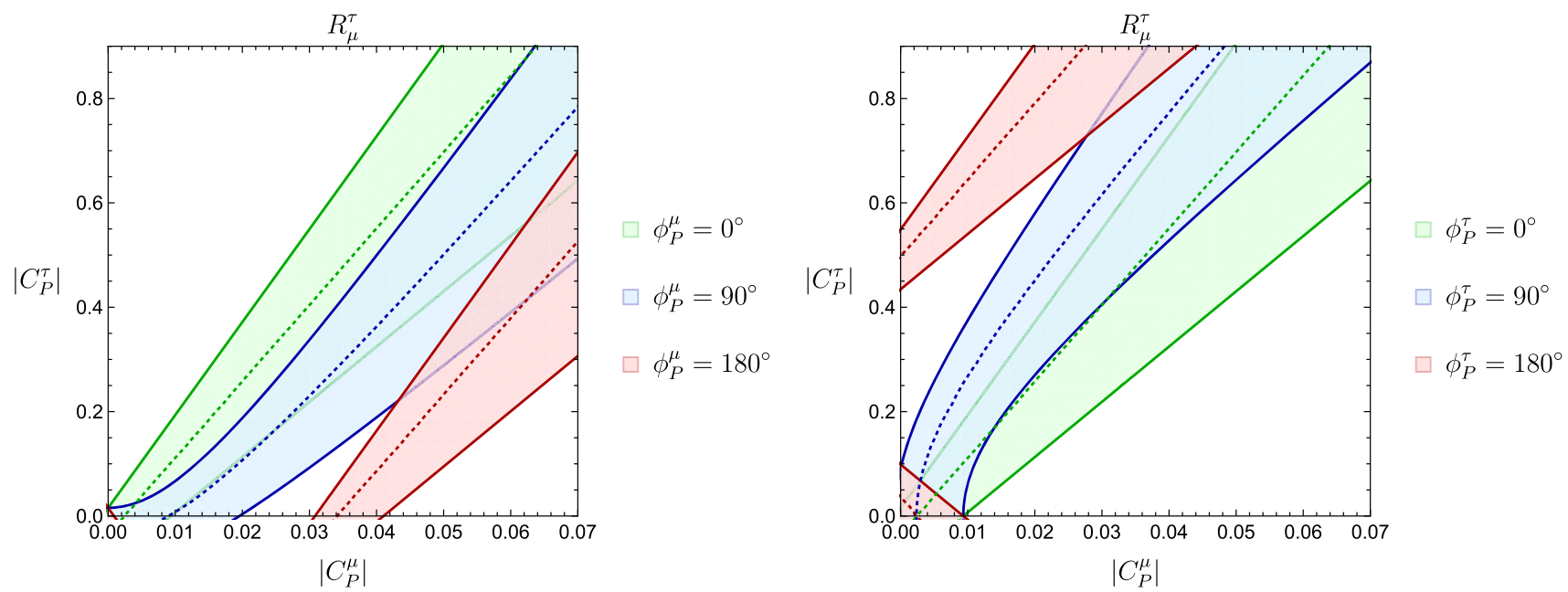

Fig. 5 Constraints in the $\left|C_{P}^{\mu}\right|-\left|C_{P}^{\tau}\right|$ plane from $R_{\mu}^{\tau}$ for different values of $\phi_{P}^{\mu}$ and $\phi_{P}^{\tau}$. In the left panel, we have $\phi_{P}^{\tau}=0^{\circ}$ while $\phi_{P}^{\mu}$ is varied, whereas in the right panel the roles of $\phi_{P}^{\mu}$ and $\phi_{P}^{\tau}$ are reversed

sum rules. For higher $q^{2}$ values, lattice determinations are available. Quark models were also used for the determination of the hadronic form factors, as discussed in Ref. [28].

The calculation of the non-perturbative contributions to $\bar{B} \rightarrow \pi$ decays is well developed. As a matter of fact, the corresponding form factors are currently known with good precision. Here we use the parameterization that extrapolates from high to low $q^{2}$ values introduced originally in Ref. [32] and discussed in more detail in Appendix A. In contrast, the form factors for the $\bar{B} \rightarrow \rho$ transitions are less precisely known, and only determinations referring independently to either the low $q^{2}$ or high $q^{2}$ regimes are available in the literature. Moreover, high $q^{2}$ calculations are more than one decade old [33] and have large uncertainties. Later in this section, we will argue on the importance of improving these results.

Semileptonic decays are used for the exclusive determination of $\left|V_{u b}\right|$, which is typically done using SM expressions. Therefore, a value of $\left|V_{u b}\right|$ based on this approach may already be affected by NP contributions. Consequently, using this parameter as an input in other NP studies may lead to wrong conclusions. To avoid this problem, we propose a different method for the determination of $\left|V_{u b}\right|$, which is described in more detail in Sect. 5. Our strategy is based on two key steps: we first obtain the NP short-distance contributions $C_{S}^{\ell}$ and $C_{P}^{\ell}$ using only ratios of branching fractions of leptonic and semileptonic processes. Then, we substitute these results in the individual expressions for the branching fractions in order to extract the value of $\left|V_{u b}\right|$.

\section{$4.1 \quad \bar{B} \rightarrow \rho \ell^{-} \bar{\nu}_{\ell}$}

Let us start our study of semileptonic decays by analyzing the processes $\bar{B}_{d}^{0} \rightarrow \rho^{+} \ell^{-} \bar{v}_{\ell}$ and $B^{-} \rightarrow \rho^{0} \ell^{-} \bar{v}_{\ell}$. To simplify the notation, we will refer to both of them as $\bar{B} \rightarrow \rho \ell^{-} \bar{v}_{\ell}$ when writing expressions that hold for both cases. Whenever a distinction is required, we will make the charges of the $B$ and $\rho$ mesons explicit. The expression for the decay width of the process $\bar{B} \rightarrow \rho \ell^{-} \bar{v}_{\ell}$ in the presence of pseudoscalar NP particles reads [34]

$$
\begin{aligned}
& \frac{d \mathcal{B}\left(\bar{B} \rightarrow \rho \ell^{-} \bar{v}_{\ell}\right)}{d q^{2}} \\
& =\frac{G_{F}^{2} \tau_{B}\left|V_{u b}\right|^{2}}{24 \pi^{3} m_{B}^{2}}\left\{\left[\frac{1}{4}\left(1+\frac{m_{\ell}^{2}}{2 q^{2}}\right)\left(H_{V,+}^{\rho 2}+H_{V,-}^{\rho 2}+H_{V, 0}^{\rho 2}\right)\right.\right. \\
& \left.\quad+\frac{3}{8} \frac{m_{\ell}^{2}}{q^{2}} H_{V, t}^{\rho 2}\right] \\
& \left.\quad+\frac{3}{8}\left|C_{P}^{\ell}\right|^{2} H_{S}^{\rho 2}+\frac{3}{4} \Re\left[C_{P}^{\ell *}\right] \frac{m_{\ell}}{\sqrt{q^{2}}} H_{S}^{\rho} H_{V, t}^{\rho}\right\} \frac{\left(q^{2}-m_{\ell}^{2}\right)^{2}}{q^{2}}\left|\vec{p}_{\rho}\right|,
\end{aligned}
$$

where $q^{2}$ is the four-momentum transfer to the leptonic system composed by the $\ell$ and the $\bar{v}_{\ell}$, which satisfies

$m_{\ell}^{2} \leq q^{2} \leq\left(M_{B}-M_{\rho}\right)^{2}$.

The hadronic form factors in the helicity basis are given by $H_{V, \pm}^{\rho}, H_{V, 0}^{\rho}, H_{V, t}^{\rho}, H_{S}^{\rho}$; more details about these quantities are provided in Appendix A. The norm of the threemomentum of the $\rho$ meson in the rest frame of the $\bar{B}$ meson is given by

$\left|\vec{p}_{\rho}\right|=\frac{\sqrt{\left[\left(M_{B}-M_{\rho}\right)^{2}-q^{2}\right]\left[\left(M_{B}+M_{\rho}\right)^{2}-q^{2}\right]}}{2 M_{B}}$.

In addition, the angular distribution contains more observables that are sensitive to (pseudo)-scalar operators. In particular, the coefficient $J_{6 c}$, which enters the forward-backward asymmetry, only takes a non-vanishing value when there are new scalar contributions [35]. For a discussion on the angular analysis of $\bar{B} \rightarrow \rho \ell^{-} \bar{v}_{\ell}$ see Ref. [24]. 
To constrain the Wilson coefficients $C_{P}^{\ell}$ (for $\ell=e, \mu, \tau$ ), we introduce the following $\left|V_{u b}\right|$-independent ratios:

$$
\begin{aligned}
\mathcal{R}_{e ; \rho}^{e} & \equiv \frac{\mathcal{B}\left(B^{-} \rightarrow e \bar{v}_{e}\right)}{\mathcal{B}\left(\bar{B} \rightarrow \rho e^{-} \bar{v}_{e}\right)}, \\
\mathcal{R}_{\mu ; \rho}^{\mu} & \equiv \frac{\mathcal{B}\left(B^{-} \rightarrow \mu^{-} \bar{v}_{\mu}\right)}{\mathcal{B}\left(\bar{B} \rightarrow \rho \mu^{-} \bar{v}_{\mu}\right)}, \\
\mathcal{R}_{\tau ; \rho}^{\tau} & \equiv \frac{\mathcal{B}\left(B^{-} \rightarrow \tau^{-} \bar{v}_{\mu}\right)}{\mathcal{B}\left(\bar{B} \rightarrow \rho \tau^{-} \bar{v}_{\tau}\right)} .
\end{aligned}
$$

Unfortunately, there is not enough experimental information available to evaluate these observables. To the best of our knowledge, in the case of the $B \rightarrow \rho$ semileptonic transitions, there are only measurements for the decay probabilities of the combined channels $\bar{B} \rightarrow \rho e^{-} \bar{v}_{e}$ and $\bar{B} \rightarrow \rho \mu^{-} \bar{v}_{\mu}$ available for different $q^{2}$ bins. In view of the recent results on lepton flavour universality violations, we urge to have independent experimental determinations for each leptonic flavour, and then assess the effects of potential NP contributions for $e^{-}, \mu^{-}$and $\tau^{-}$independently. In our study, we consider therefore the leptonic average

$$
\begin{aligned}
& \left\langle\left.\mathcal{B}\left(\bar{B}^{0} \rightarrow \rho^{+} \ell^{-} \bar{v}_{\ell}\right)\right|_{[\ell=e, \mu]}=\frac{1}{2}\left(\mathcal{B}\left(\bar{B}^{0} \rightarrow \rho^{+} e^{-} \bar{v}_{e}\right)\right.\right. \\
& \left.\quad+\mathcal{B}\left(\bar{B}^{0} \rightarrow \rho^{+} \mu^{-} \bar{v}_{\mu}\right)\right),
\end{aligned}
$$

and correspondingly for the $B^{-}$meson. In addition, we use the isospin symmetry to introduce a second average

$$
\begin{aligned}
& \left\langle\mathcal{B}\left(\bar{B} \rightarrow \rho \ell^{-} \bar{v}_{\ell}\right)\right\rangle=\frac{1}{2}\left(\left\langle\mathcal{B}\left(\bar{B}^{0} \rightarrow \rho^{+} \ell^{-} \bar{v}_{\ell}\right)\right\rangle\right. \\
& \left.\quad+2\left\langle\mathcal{B}\left(B^{-} \rightarrow \rho^{0} \ell^{-} \bar{v}_{\ell}\right)\right\rangle\right) .
\end{aligned}
$$

We start by studying the behaviour of the semileptonic decay $\bar{B} \rightarrow \rho \ell^{-} \bar{\nu}_{\ell}$ for values of $q^{2}$ within the low- $q^{2}$ range $0 \leq q^{2} \leq 12 \mathrm{GeV}^{2}$, since this is the range for which QCD sum rule calculations of the form factors are available. The experimental information provided by Belle [36] in this region leads to

$$
\begin{aligned}
& \left\langle\left.\mathcal{B}\left(\bar{B}^{0} \rightarrow \rho^{+} \ell^{-} \bar{\nu}_{\ell}\right)\right|_{[\ell=e, \mu], q^{2} \leq 12 \mathrm{GeV}^{2}}\right. \\
& =(1.90 \pm 0.20) \times 10^{-4}, \\
& 2\left\langle\mathcal{B}\left(B^{-} \rightarrow \rho^{0} \ell^{-} \bar{\nu}_{\ell}\right)\right\rangle_{[\ell=e, \mu], q^{2} \leq 12 \mathrm{GeV}^{2}} \\
& =(2.03 \pm 0.16) \times 10^{-4} .
\end{aligned}
$$

We combine the previous measurements through a weighted average [3] using the isospin symmetry as indicated in Eq. (39), yielding

$$
\begin{aligned}
& \left\langle\left.\mathcal{B}\left(\bar{B} \rightarrow \rho \ell^{-} \bar{v}_{\ell}\right)\right|_{[\ell=e, \mu], q^{2} \leq 12 \mathrm{GeV}^{2}}\right. \\
& \quad=(1.98 \pm 0.12) \times 10^{-4} .
\end{aligned}
$$

This allows us to introduce the following ratio as an alternative to the observables in Eq. (37):

$$
\begin{gathered}
\mathcal{R}_{\langle e, \mu\rangle ; \rho\left[q^{2} \leq 12\right] \mathrm{GeV}^{2}}^{\mu} \equiv \mathcal{B}\left(B^{-} \rightarrow \mu^{-} \bar{v}\right) / \\
\left\langle\left.\mathcal{B}\left(\bar{B} \rightarrow \rho \ell^{-} \bar{v}_{\ell}\right)\right|_{[\ell=e, \mu], q^{2} \leq 12 \mathrm{GeV}^{2}} .\right.
\end{gathered}
$$

Using the experimental information in Eqs. (5) and (41), we then obtain

$\mathcal{R}_{\langle e, \mu\rangle ; \rho\left[q^{2} \leq 12\right] \mathrm{GeV}^{2}}^{\mu}=(3.3 \pm 1.4) \times 10^{-3}$.

We proceed with the evaluation of the SM value. Applying the formulae in Eqs. (34), (38), (39), (42) and evaluating the corresponding form factors as indicated in Appendix A, we get

$\mathcal{R}_{\langle e, \mu\rangle ;\left.\rho\left[q^{2} \leq 12\right] \mathrm{GeV}^{2}\right|_{\mathrm{SM}} ^{\mu}}^{\mu}=(1.52 \pm 0.29) \times 10^{-3}$.

We note that this value agrees with the experimental information at the $(1-2) \sigma$ level.

To conclude this section, we would like to obtain better insights into the structure of Eq. (34). For the purpose of the discussion in the remainder of this section, it is convenient to define

$s \equiv \sqrt{q^{2}}, \quad \xi_{\ell} \equiv \frac{m_{\ell}}{s}$,

and write Eq. (34) in terms of these parameters as follows:

$$
\begin{aligned}
\frac{1}{s^{2}} & \frac{d \mathcal{B}\left(\bar{B} \rightarrow \rho \ell^{-} \bar{v}_{\ell}\right)}{d s^{2}} \\
= & \frac{G_{F}^{2} \tau_{B}\left|V_{u b}\right|^{2}}{24 \pi^{3} m_{B}^{2}}\left\{\left[\frac{1}{4}\left(1+\frac{1}{2} \xi_{\ell}^{2}\right)\left(H_{V,+}^{\rho 2}+H_{V,-}^{\rho 2}+H_{V, 0}^{\rho 2}\right)\right.\right. \\
& \left.\left.+\frac{3}{8} \xi_{\ell}^{2} H_{V, t}^{\rho 2}\right]+\frac{3}{8}\left|C_{P}\right|^{2} H_{S}^{\rho 2}+\frac{3}{4} \Re\left[C_{P}^{*}\right] \xi_{\ell} H_{S}^{\rho} H_{V, t}^{\rho}\right\} \\
& \times\left(1-\xi_{\ell}^{2}\right)^{2}\left|\vec{p}_{\rho}\right| .
\end{aligned}
$$

When $s$ is sufficiently large, we have $\xi_{\ell} \ll 1$ and we may neglect the terms proportional to $\xi_{\ell}$. We see that in this case and within the SM, only the term proportional to $H_{V,+}^{\rho 2}+$ $H_{V,-}^{\rho 2}+H_{V, 0}^{\rho 2}$ contributes to Eq. (46). It should be noted that this term is flavour universal, i.e. it does not depend on $m_{\ell}$.

One has to be careful when neglecting $\xi_{\ell}$ terms in Eq. (46) as the bounds on $q^{2}$ in Eq. (35) yield

$m_{\ell}^{2} \leq s^{2} \leq\left(M_{B}-M_{\rho}\right)^{2}$.

Consequently, at low momentum transfer, $\xi_{\ell}$ is $\mathcal{O}(1)$ and cannot be neglected. It is a priori not obvious whether Eq. (46) gives accurate results for $\xi_{\ell}=0$ when integrating over the range $0 \leq s^{2} \leq 12 \mathrm{GeV}^{2}$. In order to shed more light on this issue, we compare $\left\langle\mathcal{B}\left(\bar{B} \rightarrow \rho \ell^{-} \bar{v}_{\ell}\right)\right\rangle$ with the full rate where $\xi_{\ell} \neq 0$. To that end, we introduce

$\delta_{\mathrm{SL}}^{\rho ; \ell} \equiv \frac{\left\langle\left.\mathcal{B}\left(\bar{B} \rightarrow \rho \ell^{-} \bar{v}_{\ell}\right)\right|_{\xi_{\ell}=0}-\left\langle\mathcal{B}\left(\bar{B} \rightarrow \rho \ell^{-} \bar{v}_{\ell}\right)\right\rangle\right.}{\left\langle\mathcal{B}\left(\bar{B} \rightarrow \rho \ell^{-} \bar{v}_{\ell}\right)\right\rangle}$, 
where we integrate over the given kinematic range and take the isospin average. Assuming the SM, the numerical evaluation gives

$\delta_{\mathrm{SL}}^{\rho ; e}=4.1 \times 10^{-8}, \quad \delta_{\mathrm{SL}}^{\rho ; \mu}=3.0 \times 10^{-3}$,

thereby demonstrating that integrating Eq. (46) for $\xi_{\ell}=0$ provides a good approximation of the branching ratio for the light lepton flavours. Consequently, the assumption of flavour universality works particularly well within the SM in the case of electrons and muons. This justifies the usual approach followed for the extraction of $\left|V_{u b}\right|$ of averaging over light leptons with the aim of improving the precision by increasing the statistics. On the other hand, for $\ell=\tau$ we find

$\delta_{\mathrm{SL}}^{\rho ; \tau}=1.62$,

showing that in this case the leptonic mass cannot be neglected. This result is not surprising since the range in Eq. (47) yields

$0.16 \leq \xi_{\tau}^{2} \leq 1$,

showing how the relatively large mass of the $\tau$ has a nonnegligible impact on the phase space of the integral to calculate the semileptonic branching fraction.

\subsubsection{Constraints on pseudoscalar NP coefficients from $\bar{B} \rightarrow \rho \ell^{-} \bar{v}_{\ell}$}

Using the observable in Eq. (42) and making the assumption $C_{P}^{e}=C_{P}^{\mu}$, we can derive further constraints on the regions shown in Fig. 1. In particular, the range for $C_{P}^{\mu}$ following from $\mathcal{R}_{\langle e, \mu\rangle ; \rho\left[q^{2} \leq 12\right] \mathrm{GeV}^{2}}^{\mu}$ yields the green vertical bands shown in Fig. 6 . The combination with $R_{\mu}^{\tau}$ gives us then four allowed regions. Performing a $\chi^{2}$ fit to these two observables yields the $1 \sigma$ allowed regions given by the black

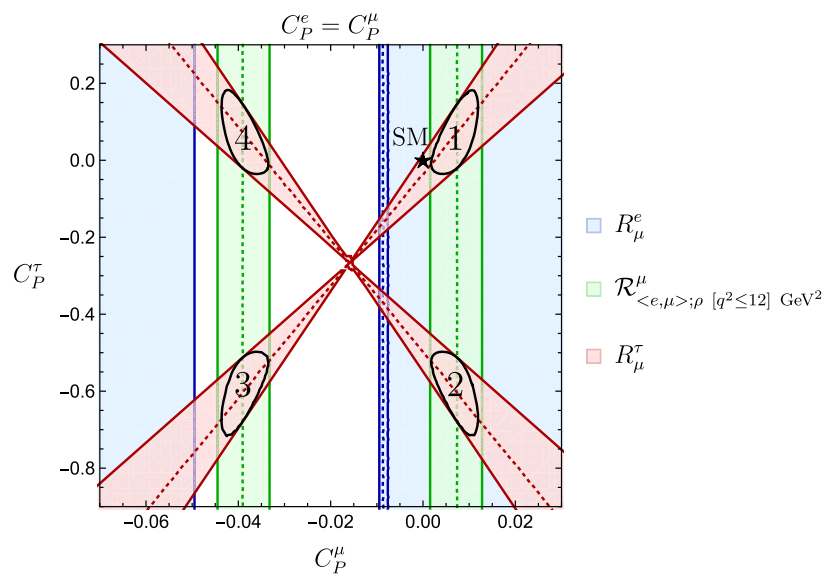

Fig. 6 Allowed regions in the $C_{P}^{\mu}-C_{P}^{\tau}$ plane utilizing the ratios $R_{\mu}^{e}$, $R_{\mu}^{\tau}$ and $\mathcal{R}_{\langle e, \mu\rangle ; \rho\left[q^{2} \leq 12\right] \mathrm{GeV}^{2}}^{\mu}$ under the assumption $C_{P}^{e}=C_{P}^{\mu}$

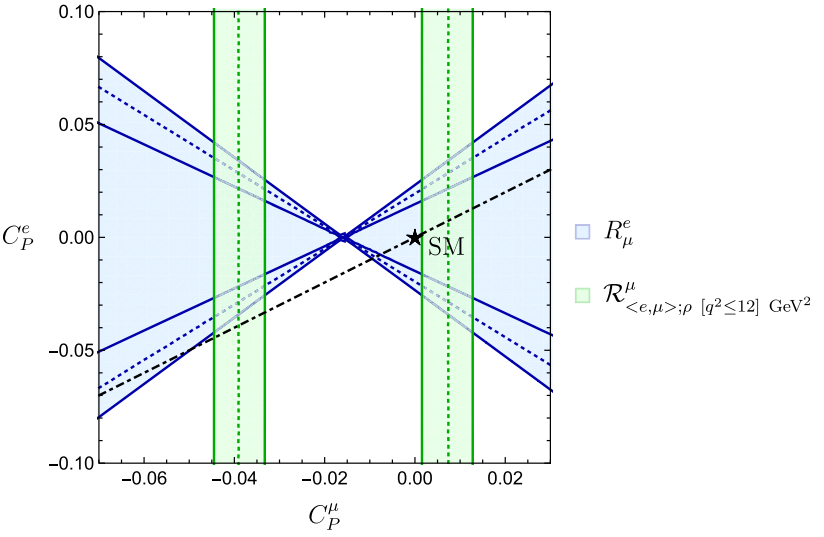

Fig. 7 Allowed regions in the $C_{P}^{\mu}-C_{P}^{e}$ plane utilizing the ratios $R_{\mu}^{e}$ and $\mathcal{R}_{\langle e, \mu\rangle ; \rho\left[q^{2} \leq 12\right] \mathrm{GeV}^{2}}^{\mu}$. The dashed-dotted line indicates the correlation arising for $C_{P}^{e}=C_{P}^{\mu}$

contours. Since the Wilson coefficients $C_{P}^{\mu}$ and $C_{P}^{e}$ are correlated, we may in addition include the ratio $R_{\mu}^{e}$ to obtain even stronger constraints. For $C_{P}^{e}=C_{P}^{\mu}$, this observable yields the blue region in Fig. 6, selecting the right green band and excluding solutions 3 and 4 satisfying $C_{P}^{\mu}<0$.

Giving up on the condition $C_{P}^{e}=C_{P}^{\mu}$, we can constrain these coefficients independently and refine the bounds in Fig. 2. We then obtain the results shown in Fig. 7, where the dashed-dotted line corresponds to $C_{P}^{e}=C_{P}^{\mu}$. We see how in both Figs. 6 and 7 the SM point is about $1 \sigma$ away from the allowed regions given by the intersection of our different constraints.

\section{$4.2 \bar{B} \rightarrow \pi \ell^{-} \bar{v}_{\ell}$}

Until now we have studied different leptonic and semileptonic constraints on the Wilson coefficient $C_{P}^{\ell}$ only. To obtain sensitivity for $C_{S}^{\ell}$, we include the processes $\bar{B}^{0} \rightarrow \pi^{+} \ell^{-} \bar{\nu}_{\ell}$ and $B^{-} \rightarrow \pi^{0} \ell^{-} \bar{v}_{\ell}$ with $\ell=e, \mu, \tau$, to be denoted generically as $\bar{B} \rightarrow \pi \ell^{-} \bar{v}_{\ell}$. The corresponding differential branching ratio in the presence of scalar NP contributions takes the following form [34]:

$$
\begin{aligned}
& \frac{d \mathcal{B}\left(\bar{B} \rightarrow \pi \ell^{-} \bar{v}_{\ell}\right)}{d q^{2}}=\frac{G_{F}^{2} \tau_{B}\left|V_{u b}\right|^{2}}{24 \pi^{3} M_{B}^{2}} \\
& \times\left\{\left[\left(1+\frac{m_{\ell}^{2}}{2 q^{2}}\right) \frac{H_{V, 0}^{\pi} 2}{4}+\frac{3}{8} \frac{m_{\ell}^{2}}{q^{2}} H_{V, t}^{\pi} 2\right]\right. \\
& \left.+\frac{3}{8}\left|C_{S}^{\ell}\right|^{2} H_{S}^{\pi}{ }^{2}+\frac{3}{4} \Re\left[C_{S}^{\ell *}\right] \frac{m_{\ell}}{\sqrt{q^{2}}} H_{S}^{\pi} H_{V, t}^{\pi}\right\} \frac{\left(q^{2}-m_{\ell}^{2}\right)^{2}}{q^{2}}\left|\vec{p}_{\pi}\right| .
\end{aligned}
$$

The full kinematical range for $q^{2}$ is

$m_{\ell}^{2} \leq q^{2} \leq\left(M_{B}-M_{\pi}\right)^{2}$.

The hadronic form factors in the helicity basis are denoted as $H_{V, 0}^{\pi}, H_{V, t}^{\pi}, H_{S}^{\pi}$ and are described in more detail in 
Appendix A. Moreover, the three momentum of the pion is given by

$\left|\vec{p}_{\pi}\right|=\frac{\sqrt{\left[\left(M_{B}-M_{\pi}\right)^{2}-q^{2}\right]\left[\left(M_{B}+M_{\pi}\right)^{2}-q^{2}\right]}}{2 M_{B}}$.

In analogy with Eq. (37), we introduce the following observables:

$$
\begin{aligned}
\mathcal{R}_{e ; \pi}^{e} & \equiv \frac{\mathcal{B}\left(B^{-} \rightarrow e \bar{v}_{e}\right)}{\mathcal{B}\left(\bar{B} \rightarrow \pi e^{-} \bar{v}_{e}\right)}, \\
\mathcal{R}_{\mu ; \pi}^{\mu} & \equiv \frac{\mathcal{B}\left(B^{-} \rightarrow \mu^{-} \bar{v}_{\mu}\right)}{\mathcal{B}\left(\bar{B} \rightarrow \pi \mu^{-} \bar{v}_{\mu}\right)}, \\
\mathcal{R}_{\tau ; \pi}^{\tau} & \equiv \frac{\mathcal{B}\left(B^{-} \rightarrow \tau^{-} \bar{v}_{\tau}\right)}{\mathcal{B}\left(\bar{B} \rightarrow \pi \tau^{-} \bar{v}_{\tau}\right)} .
\end{aligned}
$$

This set of ratios is sensitive to $C_{P}^{\ell}$ and $C_{S}^{\ell}$. Just as for the $\bar{B} \rightarrow \rho$ processes, we do not have independent determinations of the $\bar{B} \rightarrow \pi e^{-} \bar{\nu}_{e}$ and $\bar{B} \rightarrow \pi \mu^{-} \bar{v}_{\mu}$ branching ratios. Instead, the following leptonic averages are available experimentally [3]:

$$
\begin{aligned}
\left\langle\mathcal{B}\left(\bar{B}^{0} \rightarrow \pi^{+} \ell^{-} \bar{v}_{\ell}\right)\right\rangle_{[\ell=e, \mu]} & =(1.50 \pm 0.06) \times 10^{-4}, \\
2\left\langle\mathcal{B}\left(B^{-} \rightarrow \pi^{0} \ell^{-} \bar{v}_{\ell}\right)\right\rangle_{[\ell=e, \mu]} & =(1.56 \pm 0.05) \times 10^{-4} .
\end{aligned}
$$

We combine these determinations using again the isospin symmetry to obtain

$$
\left\langle\left.\mathcal{B}\left(\bar{B} \rightarrow \pi \ell^{-} \bar{v}_{\ell}\right)\right|_{[\ell=e, \mu]}=(1.53 \pm 0.04) \times 10^{-4},\right.
$$

and introduce the observable

$$
\mathcal{R}_{\langle e, \mu\rangle ; \pi}^{\mu} \equiv \mathcal{B}\left(B^{-} \rightarrow \mu^{-} \bar{v}\right) /\left\langle\left.\mathcal{B}\left(\bar{B} \rightarrow \pi \ell^{-} \bar{v}_{\ell}\right)\right|_{[\ell=e, \mu]},\right.
$$

which takes the current experimental value

$\mathcal{R}_{\langle e, \mu\rangle ; \pi}^{\mu}=(4.2 \pm 1.8) \times 10^{-3}$.

This may be compared with the SM value, for which we obtain

$\left.\mathcal{R}_{\langle e, \mu\rangle ; \pi}^{\mu}\right|_{\mathrm{SM}}=(3.18 \pm 0.96) \times 10^{-3}$,

which is in good agreement with the experimental value.

We may rewrite Eq. (52) using the parameterization introduced in Eq. (45), yielding

$$
\begin{aligned}
& \frac{1}{s^{2}} \frac{d \mathcal{B}\left(\bar{B} \rightarrow \pi \ell^{-} \bar{v}_{\ell}\right)}{d s^{2}} \\
& =\frac{G_{F}^{2} \tau_{B}\left|V_{u b}\right|^{2}}{24 \pi^{3} M_{B}^{2}}\left\{\left[\left(1+\frac{1}{2} \xi_{\ell}^{2}\right) \frac{H_{V, 0}^{\pi 2}}{4}+\frac{3}{8} \xi_{\ell}^{2} H_{V, t}^{\pi 2}\right]\right. \\
& \left.+\frac{3}{8}\left|C_{S}^{\ell}\right|^{2} H_{S}^{\pi}{ }^{2}+\frac{3}{4} \Re\left[C_{S}^{\ell *}\right] \xi_{\ell} H_{S}^{\pi} H_{V, t}^{\pi}\right\}\left(1-\xi_{\ell}^{2}\right)^{2}\left|\vec{p}_{\pi}\right| .
\end{aligned}
$$

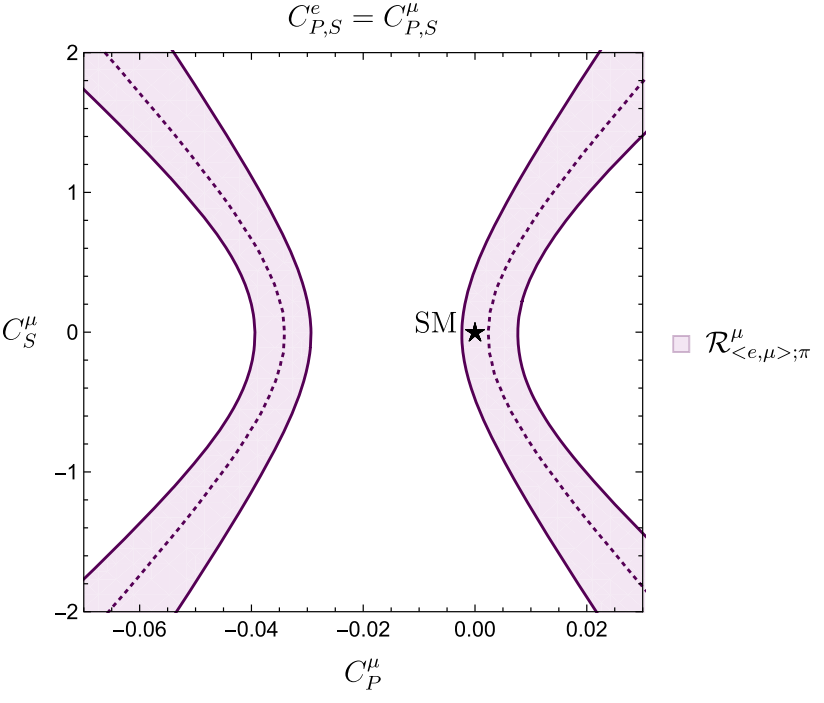

Fig. 8 Constraints in the $C_{P}^{\mu}-C_{S}^{\mu}$ plane obtained from the leptonic over semileptonic ratio $\mathcal{R}_{\langle e, \mu\rangle ; \pi}^{\mu}$

As for the $\bar{B} \rightarrow \rho$ transitions, we assess the validity of Eq. (61) through the difference

$\delta_{\mathrm{SL}}^{\pi ; \ell} \equiv \frac{\left\langle\mathcal{B}\left(\bar{B} \rightarrow \pi \ell^{-} \bar{v}_{\ell}\right)\right\rangle_{\xi_{\ell}=0}-\left\langle\mathcal{B}\left(\bar{B} \rightarrow \pi \ell^{-} \bar{v}_{\ell}\right)\right\rangle}{\left\langle\mathcal{B}\left(\bar{B} \rightarrow \pi \ell^{-} \bar{v}_{\ell}\right)\right\rangle}$,

where we consider again the isospin average. For $\delta_{\mathrm{SL}}^{\pi ; e}$ and $\delta_{\mathrm{SL}}^{\pi ; \mu}$, we find tiny values at the $10^{-8}$ and $10^{-3}$ levels, respectively, when considering the SM. This shows that taking $\xi_{\ell}=0$ in Eq. (61) provides a good approximation of the branching ratio. On the other hand, for $\ell=\tau$, the correction factor due to the mass of the $\tau$ lepton is $\delta_{\mathrm{SL}}^{\pi ; \tau}=42 \%$.

\subsubsection{Constraints on (pseudo)-scalar NP coefficients from $\bar{B} \rightarrow \pi \ell^{-} \bar{v}_{\ell}$}

Thanks to the observable in Eq. (58), we may now obtain stronger bounds for $C_{P}^{\ell}$ and $C_{S}^{\ell}$. If we make the assumptions

$C_{P}^{e}=C_{P}^{\mu}$,

$C_{S}^{e}=C_{S}^{\mu}$,

we obtain the situation shown in Fig. 8, where we notice that the SM point is included in the allowed region.

\subsection{Combining leptonic and semileptonic constraints}

We now proceed with the combination of all constraints from the different leptonic and semileptonic channels. By combining the branching fractions for the decays $\bar{B} \rightarrow \rho \ell^{-} \bar{\nu}_{\ell}$ and $\bar{B} \rightarrow \pi \ell^{-} \bar{v}_{\ell}$, we can introduce the following extra observable: 


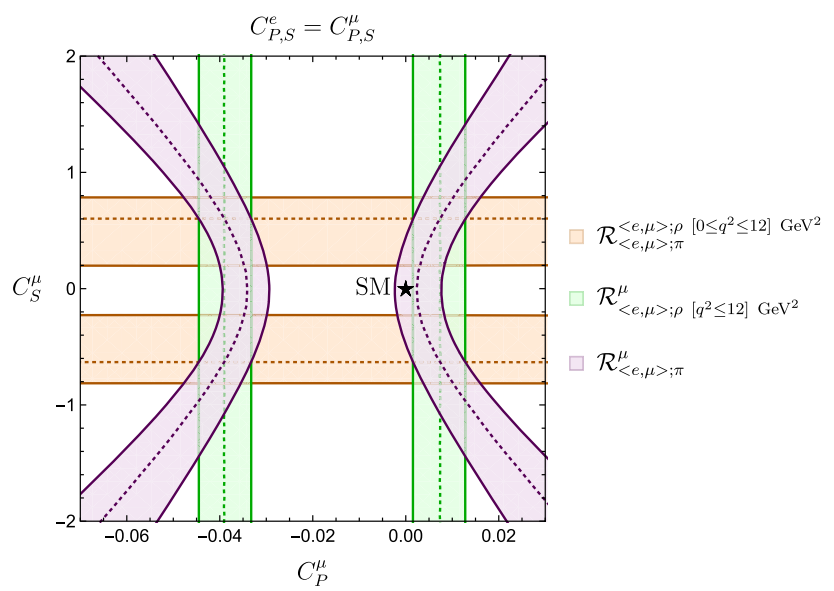

Fig. 9 Allowed regions in the $C_{P}^{\mu}-C_{S}^{\mu}$ plane considering the observables $\mathcal{R}_{\langle e, \mu\rangle ; \pi}^{\langle e, \mu\rangle ; \rho\left[0 \leq q^{2} \leq 12\right] \mathrm{GeV}^{2}}, \mathcal{R}_{\langle e, \mu\rangle ; \rho\left[q^{2} \leq 12\right] \mathrm{GeV}^{2}}^{\mu}$ and $\mathcal{R}_{\langle e, \mu\rangle ; \pi}^{\mu}$

$$
\begin{aligned}
& \mathcal{R}_{\langle e, \mu\rangle ; \pi}^{\langle e, \mu\rangle ; \rho\left[q_{\min }^{2} \leq q^{2} \leq q_{\max }^{2}\right]}=\left\langle\left.\mathcal{B}\left(\bar{B} \rightarrow \rho \ell^{-} \bar{v}_{\ell}\right)\right|_{[\ell=e, \mu]}||_{q_{\min }^{2}}^{q_{\max }^{2}}\right. \\
& \quad /\left\langle\mathcal{B}\left(\bar{B} \rightarrow \pi \ell^{-} \bar{\nu}_{\ell}\right)\right\rangle_{[\ell=e, \mu]},
\end{aligned}
$$

where the numerator is calculated by integrating the differential expression in Eq. (34) over the interval $q_{\min }^{2} \leq q^{2} \leq$ $q_{\max }^{2}$. We start by evaluating the ratio in Eq. (64) in the low$q^{2}$ regime, i.e. within the interval $q^{2} \leq 12 \mathrm{GeV}^{2}$. Therefore, using the results in Eqs. (41) and (57), we obtain

$\mathcal{R}_{\langle e, \mu\rangle ; \pi}^{\langle e, \mu\rangle ; \rho\left[0 \leq q^{2} \leq 12\right] \mathrm{GeV}^{2}}=1.29 \pm 0.09$.

Making the assumption $C_{P}^{e}=C_{P}^{\mu}$, we use the ratio in Eq. (65) to obtain stronger constraints on $C_{P}^{\mu}$ and $C_{S}^{\mu}$. The combination of observables

$\mathcal{R}_{\langle e, \mu\rangle ; \rho\left[q^{2} \leq 12\right] \mathrm{GeV}^{2}}^{\mu}, \quad \mathcal{R}_{\langle e, \mu\rangle ; \pi}^{\mu} \quad$ and $\mathcal{R}_{\langle e, \mu\rangle ; \pi}^{\langle e, \mu\rangle ; \rho\left[0 \leq q^{2} \leq 12\right] \mathrm{GeV}^{2}}$

in Eqs. (42), (58) and (64), respectively, leads to the regions shown in Fig. 9. Interestingly, the semileptonic over semileptonic ratio defines two horizontal bands that exclude the SM point by $(1-2) \sigma$.

The tension with the SM found in Fig. 9 is an interesting effect that we proceed to investigate in more detail. To this end, we consider the partition of the interval $0 \leq$ $q^{2} \leq 12 \mathrm{GeV}^{2}$ given in Table 1. Calculating the observable $\mathcal{R}_{\langle e, \mu\rangle ; \pi}^{\langle e, \mu\rangle ; \rho}$ in each subinterval yields

$$
\mathcal{R}_{\langle e, \mu\rangle ; \pi}^{\langle e, \mu\rangle ; \rho\left[0 \leq q^{2} \leq 4\right] \mathrm{GeV}^{2}}=0.31 \pm 0.05
$$

$$
\begin{gathered}
\mathcal{R}_{\langle e, \mu\rangle ; \pi}^{\langle e, \mu\rangle ; \rho\left[4 \leq q^{2} \leq 8\right] \mathrm{GeV}^{2}}=0.50 \pm 0.05 \\
\mathcal{R}_{\langle e, \mu\rangle ; \pi}^{\langle e, \mu\rangle ; \rho\left[8 \leq q^{2} \leq 12\right] \mathrm{GeV}^{2}}=0.47 \pm 0.05
\end{gathered}
$$

We present the constraints from these observables in Fig. 10. We observe that the SM point is excluded within the subintervals $[0,4] \mathrm{GeV}^{2}$ and $[8,12] \mathrm{GeV}^{2}$. However, it is contained within $[4,8] \mathrm{GeV}^{2}$. Thus, we can now identify the source of the tension with the SM point found in Fig. 9.

In view of the tension with the SM found in Figs. 9 and 10, we investigate whether this effect persists for $12 \mathrm{GeV}^{2} \leq q^{2}$. For high $q^{2}$ values, the theoretical determination of the form factors is done through lattice calculations. To the best of our knowledge, the most recent determination of the $\bar{B} \rightarrow \rho$ form factors available is discussed in Ref. [33], where the range

$$
12.7 \mathrm{GeV}^{2} \leq q^{2} \leq 18.2 \mathrm{GeV}^{2}
$$

is considered. It should be noted that this reference is more than 14 years old. Moreover, there is not an analytical parameterization of the form factors similar to the one for the low $-q^{2}$ regime presented in Appendix A. Consequently, we extract the required information directly from the distributions presented in Ref. [33] that have large errors. In the absence of analytical expressions for the form factors, we run the risk of over estimating the uncertainties associated with the branching fraction $\mathcal{B}\left(\bar{B} \rightarrow \rho \ell^{-} \bar{v}_{\ell}\right)$. We can avoid this problem by using the differential branching ratio $d \mathcal{B}\left(B^{-} \rightarrow \rho^{0} \ell^{-} \bar{v}_{\ell}\right) / d q^{2}$ at specific values of $q^{2}$ presented in Eq. (34). For this part of the analysis, we cannot use isospin-averaged quantities because the experimental partition for $\mathcal{B}\left(\bar{B}_{d}^{0} \rightarrow \rho^{+} \ell^{-} \bar{\nu}_{\ell}\right)$ cannot be compared against the corresponding theoretical range given by the form factors. Therefore we restrict ourselves to the decay channel $B^{-} \rightarrow \rho^{0} \ell^{-} \bar{v}_{\ell}$ and consider the following observable:

$d \mathcal{R}_{\langle e, \mu\rangle ; \pi}^{\langle e, \mu\rangle ; \rho}=\frac{2\left\langle d \mathcal{B}\left(B^{-} \rightarrow \rho^{0} \ell^{-} \bar{v}_{\ell}\right) / d q^{2}\right\rangle_{[\ell=e, \mu]}}{\left\langle\mathcal{B}\left(\bar{B} \rightarrow \pi \ell^{-} \bar{v}_{\ell}\right)\right\rangle_{[\ell=e, \mu]}}$

In Ref. [33], two different determinations of the form factors are available depending on the value of the coupling constant $\beta=6 / g_{0}^{2}$. In particular, we have $\beta=6.0$ and $\beta=6.2$. Moreover, the available experimental data allow us to evaluate the numerator in Eq. (68) at $q^{2}=15 \mathrm{GeV}^{2}$ and $q^{2}=17 \mathrm{GeV}^{2}$, yielding
Table 1 Experimental values of $\mathcal{B}\left(\bar{B} \rightarrow \rho \ell^{-} \bar{v}_{\ell}\right)$ in different $q^{2}$ intervals [36]. The fourth column gives the isospin averages of the values in the second and third columns

\begin{tabular}{llll}
\hline$\Delta q^{2}\left(\mathrm{GeV}^{2}\right)$ & $2\left\langle\mathcal{B}\left(B^{-} \rightarrow \rho^{0} \ell^{-} \bar{\nu}_{\ell}\right)\right\rangle_{[\ell=e, \mu]}$ & $\left\langle\mathcal{B}\left(\bar{B}^{0} \rightarrow \rho^{+} \ell^{-} \bar{v}_{\ell}\right)\right\rangle_{[\ell=e, \mu]}$ & $\left\langle\mathcal{B}\left(\bar{B} \rightarrow \rho \ell^{-} \bar{v}_{\ell}\right)\right\rangle_{[\ell=e, \mu]}$ \\
\hline$[0,4]$ & $(5.54 \pm 0.92) \times 10^{-5}$ & $(3.73 \pm 1.06) \times 10^{-5}$ & $(4.76 \pm 0.69) \times 10^{-5}$ \\
{$[4,8]$} & $(7.92 \pm 0.96) \times 10^{-5}$ & $(7.18 \pm 1.16) \times 10^{-5}$ & $(7.62 \pm 0.74) \times 10^{-5}$ \\
{$[8,12]$} & $(6.84 \pm 0.89) \times 10^{-5}$ & $(8.06 \pm 1.23) \times 10^{-5}$ & $(7.26 \pm 0.72) \times 10^{-5}$
\end{tabular}



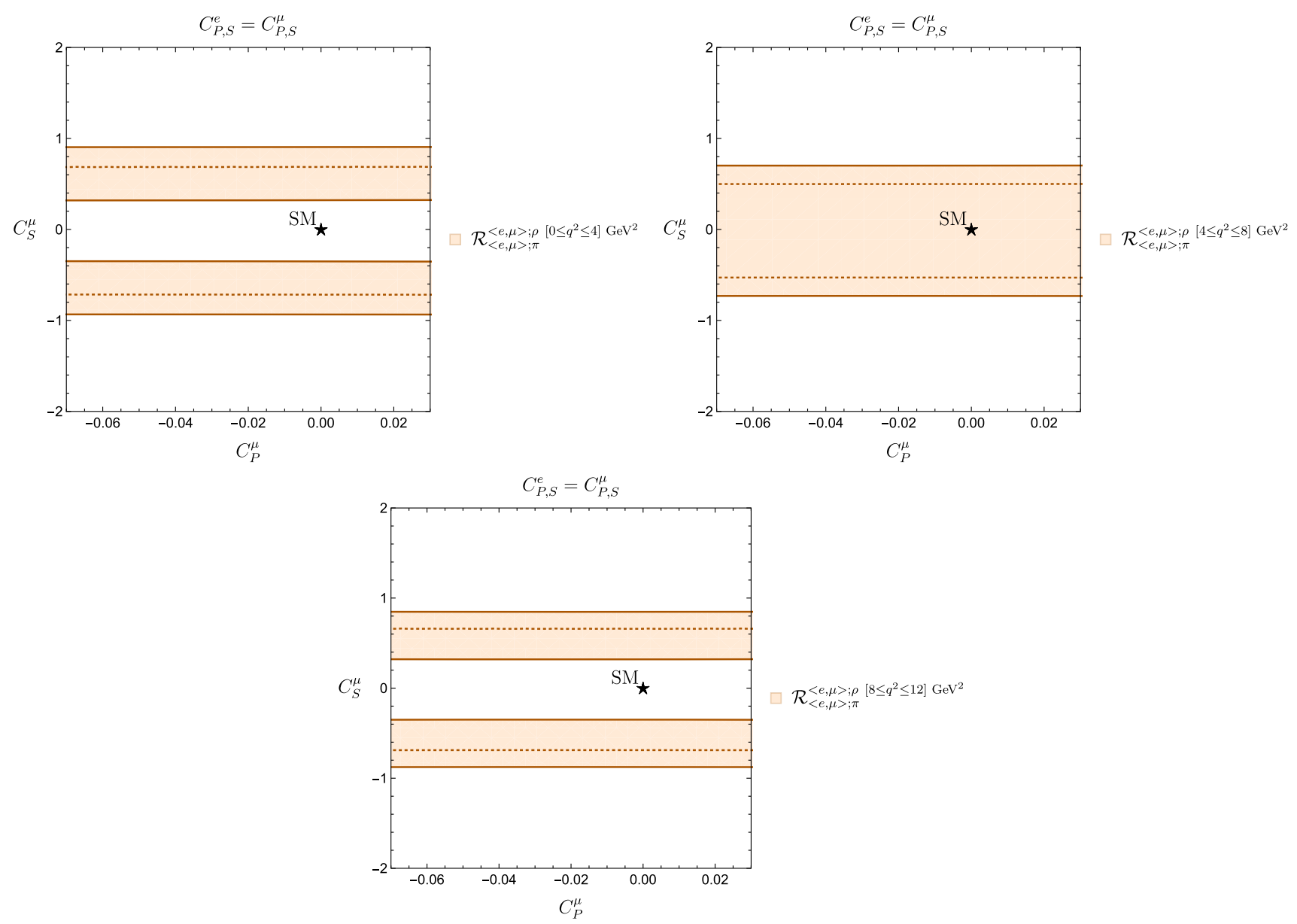

Fig. 10 Constraints from $\mathcal{R}_{\langle e, \mu\rangle ; \pi}^{\langle e, \mu\rangle ; \rho}$ in the $C_{P}^{\mu}-C_{S}^{\mu}$ plane for different intervals of $q^{2}$

$$
\begin{aligned}
& \left.d \mathcal{R}_{\langle e, \mu\rangle ; \pi}^{\langle e, \mu\rangle ; \rho}\right|_{q^{2}=15 \mathrm{GeV}^{2}}=0.14 \pm 0.02,\left.\quad d \mathcal{R}_{\langle e, \mu\rangle ; \pi}^{\langle e, \mu\rangle ; \rho}\right|_{q^{2}=17 \mathrm{GeV}^{2}} \\
& =0.11 \pm 0.02
\end{aligned}
$$

The corresponding plots are shown in Fig. 11 for $\beta=6.0$ and in Fig. 12 for $\beta=6.2$.

Just as for the low $-q^{2}$ region, a small tension with the SM appears in the case of $\beta=6.0$ with $q^{2}=17 \mathrm{GeV}^{2}$. However, a more precise determination of the form factors in the high$q^{2}$ regime is required in order to understand the origin of this discrepancy: it can certainly be triggered by the theoretical precision of the non-perturbative contributions. Indeed, the study presented in [33] was performed when the lattice calculations technology was in its early stages of development and an underestimation of the uncertainties cannot be discarded. A very interesting prospect would be the presence of NP; this possibility is quite exciting and is in principle allowed by the theoretical and experimental information available at the moment. In addition, an interpolation between the lowand high $-q^{2}$ regimes for the $B \rightarrow \rho$ transitions will allow a full use of the experimental determinations.

\subsection{CP violation}

Finally, we would like to study the implications of CPviolating phases once we combine the different leptonic and semileptonic constraints described at the beginning of this Section and in Sect. 3. Since the direct CP asymmetries defined in Eq. (10) would take essentially vanishing values for the (semi)leptonic decays, we follow the approach introduced in Sect. 3.2 for leptonic processes and explore the implications of new CP-violating phases in the short distance contributions, i.e. complex Wilson coefficients. Specifically, we analyse correlations between the norms and phases of the short-distance contributions, as well as between norms of different coefficients.

To begin with, we consider the constraints in the $\phi_{P}^{\mu}$ $\left|C_{P}^{\mu}\right|$ plane shown in Fig. 4. This analysis was performed under the assumption $C_{P}^{e}=C_{P}^{\mu}$ using only the observable $R_{\mu}^{e}$. We complement this study by including the ratio $\mathcal{R}_{\langle e, \mu\rangle ; \rho\left[q^{2} \leq 12\right] \mathrm{GeV}^{2}}^{\mu}$, introduced in Eq. (42). The new regions are shown in Fig. 13. We notice that the SM point falls within the allowed regions. Additionally, the norm of 

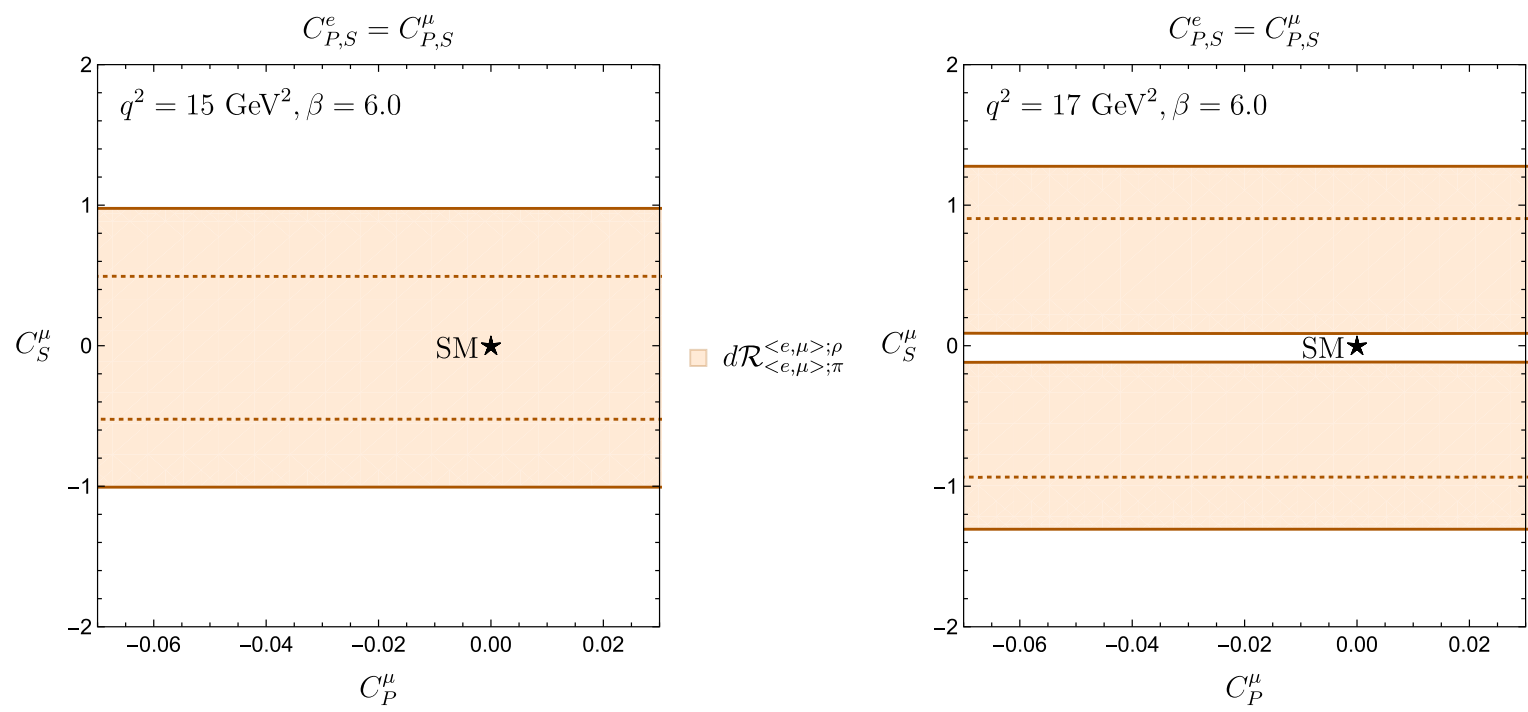

$d \mathcal{R}_{<e, \mu>; \pi}^{<e, \mu>; \rho}$

Fig. 11 Regions in the $C_{P}^{\mu}-C_{S}^{\mu}$ plane allowed by the observable $d \mathcal{R}_{\langle e, \mu\rangle ; \pi}^{\langle e, \mu\rangle ; \rho}$ for $q^{2}=15 \mathrm{GeV}^{2}$ (left) and $q^{2}=17 \mathrm{GeV}^{2}$ (right), considering $\beta=6.0$
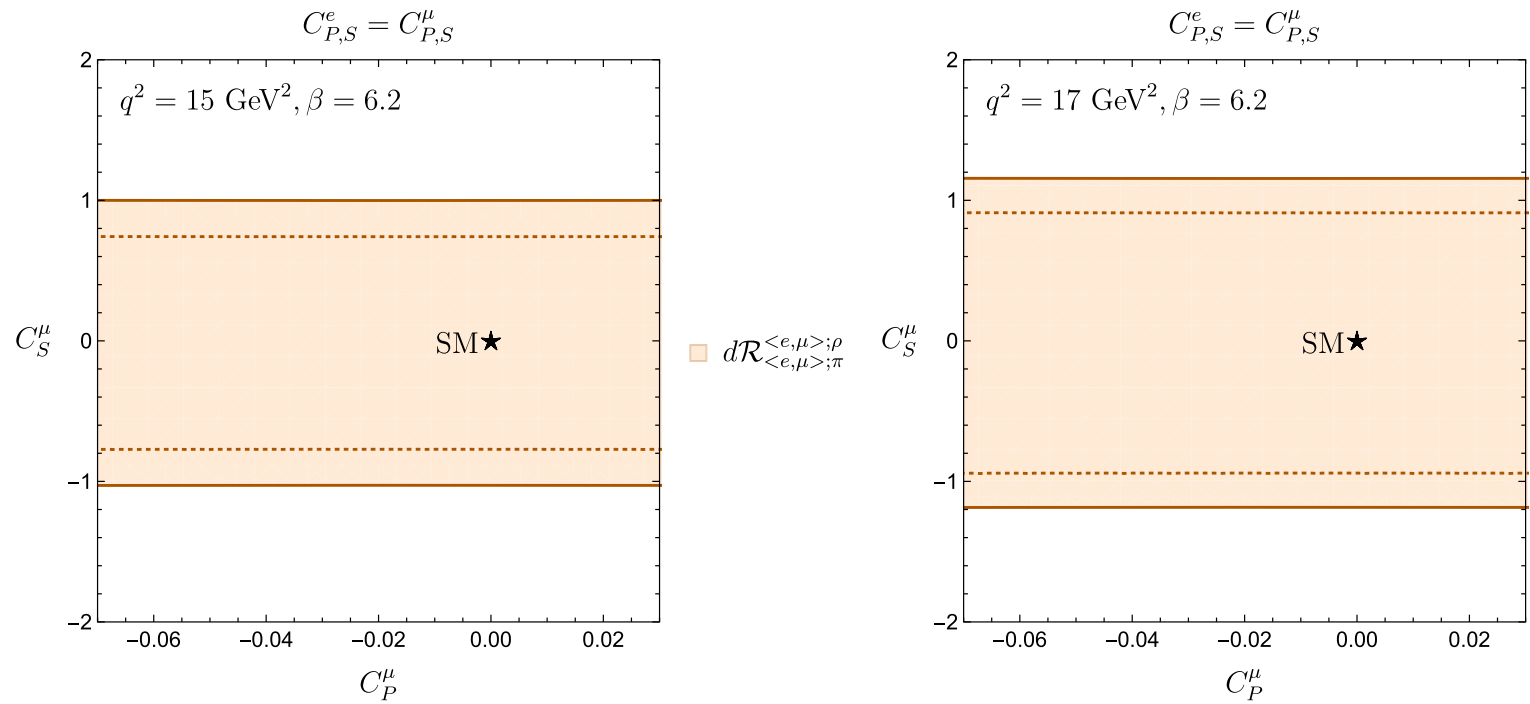

$d \mathcal{R}_{<e, \mu>; \pi}^{<e, \mu>\rho}$

Fig. 12 Regions in the $C_{P}^{\mu}-C_{S}^{\mu}$ plane allowed by the observable $d \mathcal{R}_{\langle e, \mu\rangle ; \pi}^{\langle e, \mu\rangle ; \rho}$ for $q^{2}=15 \mathrm{GeV}^{2}$ (left) and $q^{2}=17 \mathrm{GeV}^{2}$ (right), considering $\beta=6.2$

the pseudo-scalar Wilson coefficient is bounded, at the one sigma level this bound reads

$\left|C_{P}^{\mu}\right| \leq 0.042$

We continue by adding the observable $\mathcal{R}_{\langle e, \mu\rangle ; \rho\left[q^{2} \leq 12\right] \mathrm{GeV}^{2}}^{\mu}$ to the analysis shown in Fig. 5; to incorporate this observable we assume $C_{P}^{e}=C_{P}^{\mu}$. We explore the correlations between $\left|C_{P}^{\mu}\right|-\left|C_{P}^{\tau}\right|$ considering different values for the phases $\phi_{P}^{\mu}$ and $\phi_{P}^{\tau}$. We first fix $\phi_{P}^{\mu}=0^{\circ}$ and allow $\phi_{P}^{\tau}$ to change in steps of $45^{\circ}$ up to the value $\phi_{P}^{\tau}=180^{\circ}$. The resulting patterns are shown in Fig. 14, where the overlapping region of the two constraints is indicated in blue. We can see how the regions evolve along the vertical direction. Once the value
$\phi_{P}^{\tau}=180^{\circ}$ is reached, the behaviour is cyclic and the resulting patterns come back into themselves.

Finally, we allow $\phi_{P}^{\mu}$ to change as well. Unlike the previous case, the evolution is now along the horizontal direction. By scanning $\phi_{P}^{\mu}$ and $\phi_{P}^{\tau}$ within the interval $\left[0^{\circ}, 180^{\circ}\right]$ we generate the smeared plot shown in Fig. 15. We have highlighted the steps corresponding to: $\left(\phi_{P}^{\mu}=0^{\circ}, \phi_{P}^{\tau}=0^{\circ}\right)$, $\left(\phi_{P}^{\mu}=0^{\circ}, \phi_{P}^{\tau}=180^{\circ}\right),\left(\phi_{P}^{\mu}=180^{\circ}, \phi_{P}^{\tau}=0^{\circ}\right)$ and $\left(\phi_{P}^{\mu}=180^{\circ}, \phi_{P}^{\tau}=180^{\circ}\right)$.

\section{Determination of $\left|V_{u b}\right|$}

The extraction of $\left|V_{u b}\right|$ from semileptonic decays is usually done under the assumption of the SM, although NP contribu- 


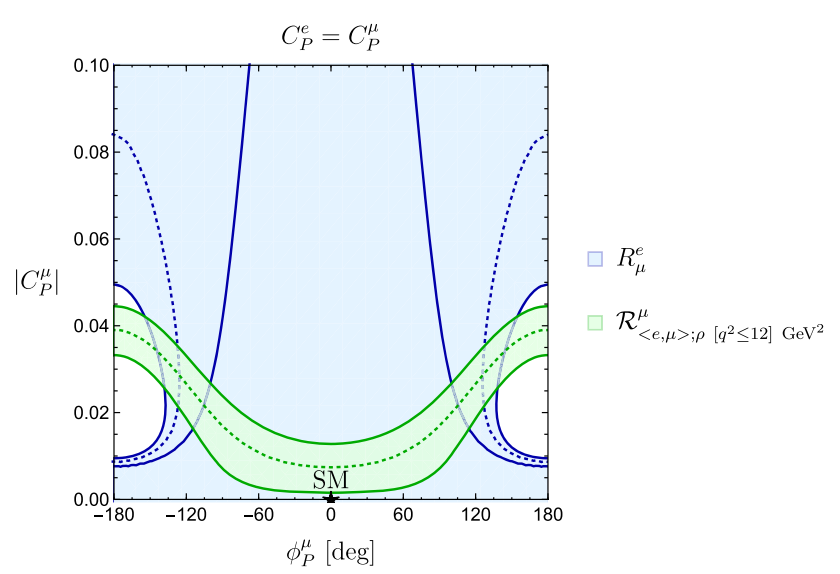

Fig. 13 Regions obtained in the $\phi_{P}^{\mu}-\left|C_{P}^{\mu}\right|$ plane from $R_{\mu}^{e}$ and $\mathcal{R}_{\langle e, \mu\rangle ; \rho\left[q^{2} \leq 12\right] \mathrm{GeV}^{2}}^{\mu}$, assuming universality for the light lepton flavours

tions may also have an impact [25]. For instance, the effect of a new right-handed vector current on the determination of $\left|V_{u b}\right|$ has been discussed in Ref. [24], where also new ways to search for such NP effects using $\bar{B} \rightarrow \rho \ell^{-} \bar{v}_{\ell}$ decays are presented. Here we provide a general strategy that allows us to determine $\left|V_{u b}\right|$ in the presence of new scalar and pseudoscalar contributions. We remind ourselves that the branching fractions of the leptonic decays and the semileptonic $\bar{B} \rightarrow \rho \ell^{-} \bar{v}_{\ell}$ transitions are only sensitive to the pseudoscalar NP operator. On the other hand, $\mathcal{B}\left(\bar{B} \rightarrow \pi \ell^{-} v\right)$ depends exclusively on the scalar Wilson coefficient. Throughout this section and Sect. 6, we consider only the range $0 \leq q^{2} \leq 12$ $\mathrm{GeV}^{2}$ for the $\bar{B} \rightarrow \rho \ell^{-} \bar{v}_{\ell}$ transition and therefore we omit this information from the labels of the ratios. Consequently, unless stated otherwise, we take

$\mathcal{R}_{\langle e, \mu\rangle ; \rho}^{\mu} \equiv \mathcal{R}_{\langle e, \mu\rangle ; \rho\left[q^{2} \leq 12\right] \mathrm{GeV}^{2}}^{\mu}$.

We start our discussion by focussing our attention on observables containing only the Wilson coefficient $C_{P}^{\ell}$. Moreover, we will assume universal scalar and pseudoscalar interactions for light leptons, i.e. $C_{S}^{e}=C_{S}^{\mu}, C_{P}^{e}=C_{P}^{\mu}$. There are then two key steps to obtain $\left|V_{u b}\right|$ that can be summarized as follows:

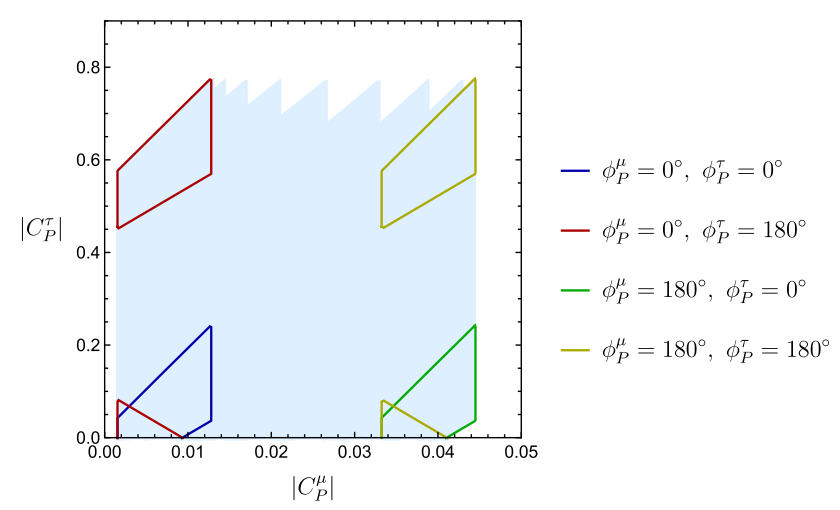

Fig. 15 Region in the $\left|C_{P}^{\mu}\right|-\left|C_{P}^{\tau}\right|$ plane obtained from the overlap between the contours following from $R_{\mu}^{\tau}$ and $\mathcal{R}_{\langle e, \mu\rangle ; \rho\left[q^{2} \leq 12\right] \mathrm{GeV}^{2}}^{\mu}$. We vary the phases $\phi_{P}^{\mu}$ and $\phi_{P}^{\tau}$ independently in the interval $\left[0^{\circ}, 180^{\circ}\right]$, giving the blue background region. The jagged upper boundary is due to varying $\phi_{P}^{\mu}$ in steps of $20^{\circ}$. The coloured regions indicate solutions obtained for $\phi_{P}^{\mu}, \phi_{P}^{\tau} \in\left\{0^{\circ}, 180^{\circ}\right\}$

1. Perform a $\left|V_{u b}\right|$-independent extraction of $C_{P}^{\ell}$. This can be achieved using the ratios introduced in Sects. 3 and 4.

2. Substitute the ranges for $C_{P}^{\ell}$ in any of the leptonic or semileptonic branching ratios available, i.e. $\mathcal{B}\left(B^{-} \rightarrow\right.$ $\left.\mu^{-} \bar{v}_{\mu}\right)$ or $\mathcal{B}\left(\bar{B} \rightarrow \rho \ell^{-} \bar{v}_{\ell}\right)$, and then solve for $\left|V_{u b}\right|$.

This procedure can be implemented in different ways employing the constraints discussed in the previous sections. For instance, we can use the bounds for the pseudoscalar NP short-distance contributions derived in Sects. 3, 4 and presented in Fig. 6. One of the problems with this approach is that possible correlations between the observables are not taken into account. Let us now elaborate on an alternative strategy which avoids this issue:

- Using the expressions for $\mathcal{R}_{\langle e, \mu\rangle ; \rho}^{\mu}$ introduced in Eq. (42), we solve for $C_{P}^{\mu}$. Since we are assuming universal NP contributions for electrons and muons, this ratio depends only on one single NP coefficient.

- The previous step leads to the function $C_{P}^{\mu}\left(\mathcal{R}_{\langle e, \mu\rangle ; \rho}^{\mu}\right)$. There are two solutions satisfying independently $C_{P}^{\mu}<0$ and $0<C_{P}^{\mu}$. Looking at Fig. 6, we see that only $0<C_{P}^{\mu}$ is consistent with all the available constraints.
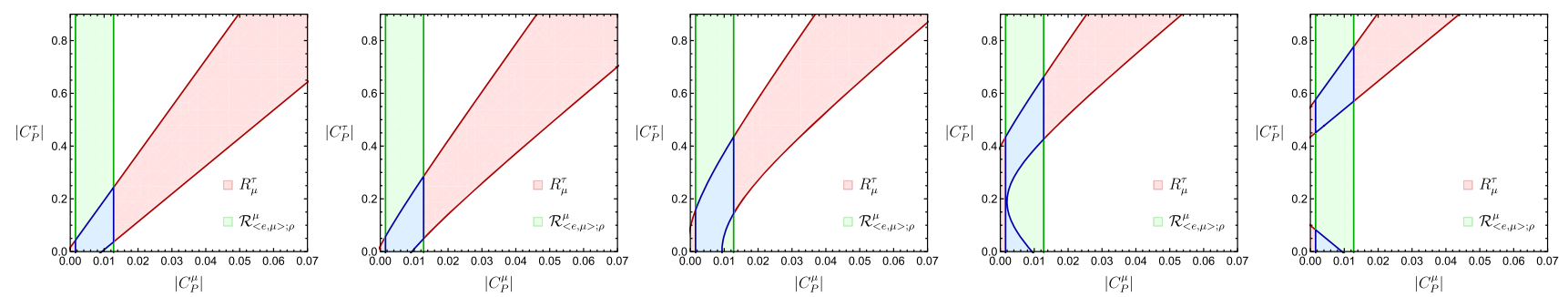

Fig. 14 Evolution of the $\left|C_{P}^{\mu}\right|-\left|C_{P}^{\tau}\right|$ plane for $\phi_{P}^{\mu}=0^{\circ}$ and different values of $\phi_{P}^{\tau}$. The first and the last plots correspond to $\phi_{P}^{\tau}=0^{\circ}$ and $\phi_{P}^{\tau}=180^{\circ}$, respectively, whereas the plots in between show increasing values of $\phi_{P}^{\tau}$ in steps of $45^{\circ}$ from left to right 


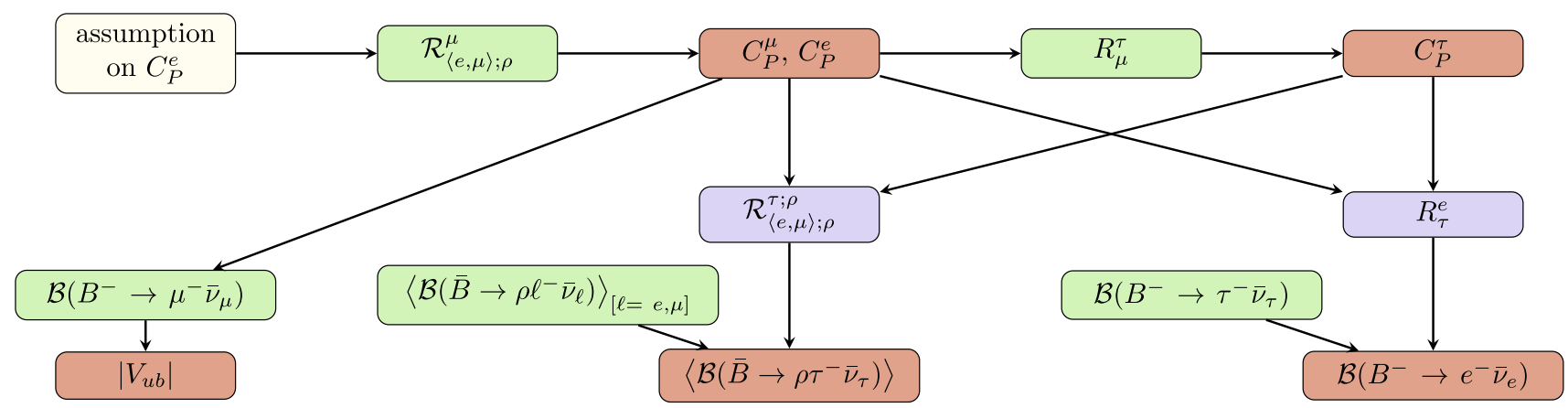

Fig. 16 Flowchart illustrating the strategies for the determination of $\left|V_{u b}\right|$ and the predictions of $\left\langle\mathcal{B}\left(\bar{B} \rightarrow \rho \tau^{-} \bar{v}_{\tau}\right)\right\rangle$ and $\mathcal{B}\left(B^{-} \rightarrow e^{-} \bar{v}_{e}\right)$

- Finally, we evaluate any of the individual branching fractions $\mathcal{B}\left(B^{-} \rightarrow \mu^{-} \bar{v}_{\mu}\right)$ or $\left\langle\left.\mathcal{B}\left(\bar{B} \rightarrow \rho \ell^{-} \bar{v}_{\ell}\right)\right|_{[\ell=e, \mu]}\right.$ in the interval for $C_{P}^{\mu}$ obtained above. From the resulting expression, we can determine the only unknown left: the value of $\left|V_{u b}\right|$.

This strategy has been summarized in the flowchart in Fig. 16.

Up to now we have shown how it is possible to extract $\left|V_{u b}\right|$ from observables involving $C_{P}^{\mu}$. We can, however, incorporate also the constraints for $C_{P}^{\tau}$. With this in mind, we consider $R_{\mu}^{\tau}$ defined in Eq. (19), which depends both on $C_{P}^{\mu}$ and on $C_{P}^{\tau}$. We reduce the number of independent parameters by substituting $C_{P}^{\mu}\left(\mathcal{R}_{\langle e, \mu\rangle ; \rho}^{\mu}\right)$ in $R_{\mu}^{\tau}$. The resulting expression will depend only on $C_{P}^{\tau}$ and can be inverted to obtain this coefficient as a function of $R_{\mu}^{\tau}$ and $\mathcal{R}_{\langle e, \mu\rangle ; \rho}^{\mu}$, which can then be inserted into $\mathcal{B}\left(B^{-} \rightarrow \tau^{-} \bar{\nu}_{\tau}\right)$ to extract $\left|V_{u b}\right|$.

Following any of the two methods described above leads to consistent results. This is actually not surprising since by adding $\mathcal{B}\left(B^{-} \rightarrow \tau^{-} \bar{\nu}_{\tau}\right)$ to our set of observables we are also including an additional coefficient $C_{P}^{\tau}$. The result will be the same if we consider ratios containing $\left\langle\left.\mathcal{B}\left(\bar{B} \rightarrow \pi \ell^{-} \bar{v}_{\ell}\right)\right|_{[\ell=e, \mu]}\right.$, which bring $C_{S}^{\mu}$ as an extra parameter into the analysis.

Following any of the procedures described above, we find for the universal scenario

$\left|V_{u b}\right|=(3.31 \pm 0.32) \times 10^{-3}$.

This result is in agreement with the CKMFitter value in Eq. (3) but the latter is three times more precise. However, our aim is to illustrate how to account properly for NP effects during the determination of $\left|V_{u b}\right|$.

We may also relax the universality condition for the light leptons. However, in order to use the experimental result in Eq. (41), we have to make an assumption on the correlation between $C_{P}^{e}$ and $C_{P}^{\mu}$. Here we consider four scenarios:

1. $C_{P}^{e} \ll C_{P}^{\mu}$; in particular, we explore

$$
C_{P}^{e}=(1 / 10) C_{P}^{\mu} \text {. }
$$

2. $C_{P}^{\mu} \ll C_{P}^{e}$; we focus on

$$
C_{P}^{e}=10 C_{P}^{\mu}
$$

3. The 2HDM, where according to Eq. (16), we have

$$
C_{P}^{e}=\frac{m_{e}}{m_{\mu}} C_{P}^{\mu}, \quad C_{P}^{\tau}=\frac{m_{\tau}}{m_{\mu}} C_{P}^{\mu} .
$$

4. NP entering only through the third generation:

$$
C_{P}^{\tau} \neq 0, \quad C_{P}^{e}=C_{P}^{\mu}=0 .
$$

Let us consider first the cases $C_{P}^{e}=(1 / 10) C_{P}^{\mu}$ and $C_{P}^{e}=$ $10 C_{P}^{\mu}$. After imposing the relevant leptonic and semileptonic constraints, we obtain the plots shown in Fig. 17. From the left plot, we see how for $C_{P}^{e}=(1 / 10) C_{P}^{\mu}$ the four regions lying in the intersections of the observables $\mathcal{R}_{\langle e, \mu\rangle ; \rho}^{\mu}$ and $R_{\mu}^{\tau}$ are allowed. They are enclosed by four ellipses shown in the plot and numbered clockwise starting with the one in the upper-right corner. We obtain $\left|V_{u b}\right|$ by applying the methods described at the beginning of this section, and summarize our results in the second column of Table 2.

If we consider the correlation $C_{P}^{e}=10 C_{P}^{\mu}$, we obtain the right plot in Fig. 17, where the observable $R_{\mu}^{e}$ selects two narrow vertical sections inside the two ellipses on the right. As for $C_{P}^{e}=(1 / 10) C_{P}^{\mu}$, the numerical results are summarized in Table 2.

For the 2HDM the only relevant constraint is given by $\mathcal{R}_{\langle e, \mu\rangle ; \rho}^{\mu}$. According to Eq. (75), all the Wilson coefficients depend only on $C_{P}^{\mu}$. Using the corresponding experimental information, we may solve for this coefficient, yielding

$C_{P}^{\mu}=-0.0391 \pm 0.0055 \quad \vee \quad C_{P}^{\mu}=0.0074 \pm 0.0054$.

Finally, in our 4th scenario, NP enters exclusively though $C_{P}^{\tau}$. Therefore the only useful constraint is given by $R_{\mu}^{\tau}$. Using the experimental determination in Eq. (21) leads to the following two solutions: 

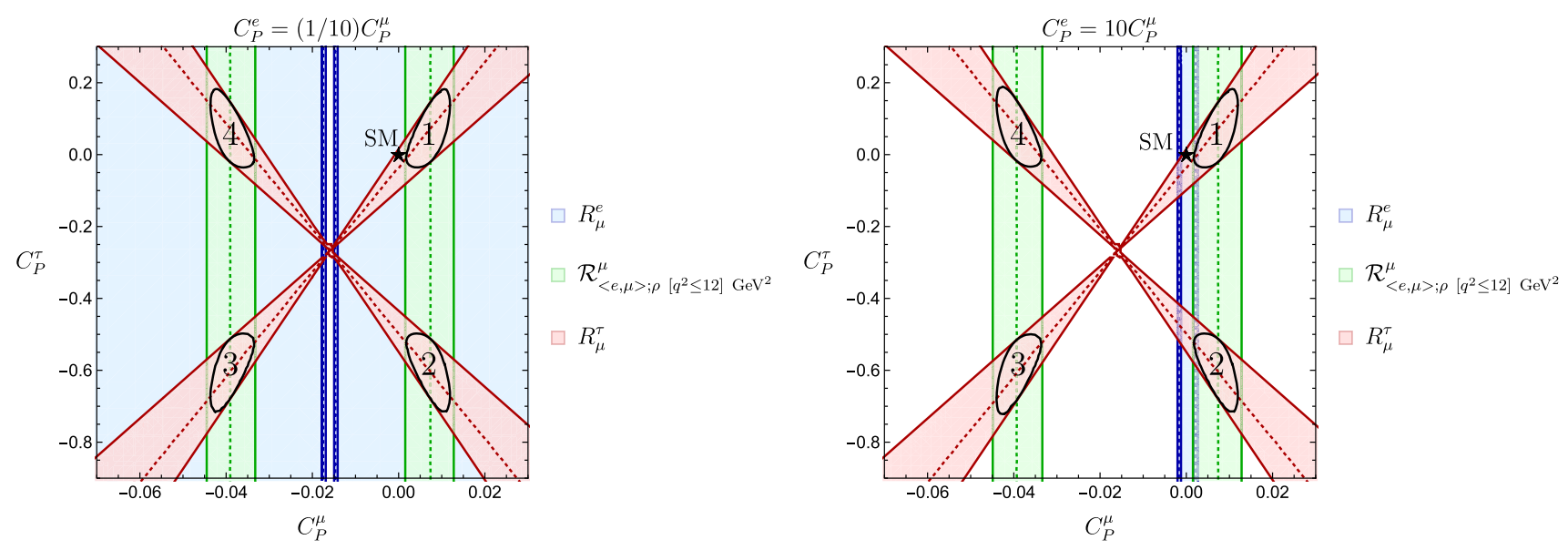

Fig. 17 Regions allowed for $C_{P}^{\mu}$ and $C_{P}^{\tau}$ for the scenarios discussed in the text

Table 2 Summary of the determination of $\left|V_{u b}\right|$ and the predictions for $\mathcal{B}\left(B^{-} \rightarrow e^{-} \bar{v}_{e}\right)$ and $\left\langle\mathcal{B}\left(\bar{B} \rightarrow \rho \tau^{-} \bar{\nu}_{\tau}\right)\right\rangle$ in the different scenarios discussed in the text

$C_{P}^{\tau}=-0.499 \pm 0.056 \quad \vee \quad C_{P}^{\tau}=-0.034 \pm 0.055$.

The resulting values for $\left|V_{u b}\right|$ are summarized in Table 2.

For most of these studies, the values of $\left|V_{u b}\right|$ coincide with one another at the level of the significant digits. However, in the scenario where the NP enters only through the third generation, our numerical result for $\left|V_{u b}\right|$ is higher in comparison with the other cases. In this respect it agrees with the inclusive $\left|V_{u b}\right|$ determinations. This is certainly an interesting observation, although the uncertainty is still too large to draw any further conclusions.

\section{Predictions of branching ratios}

Here we provide predictions for branching ratios which have not yet been measured:
$\mathcal{B}\left(B^{-} \rightarrow e^{-} \bar{\nu}_{e}\right), \quad \mathcal{B}\left(\bar{B} \rightarrow \rho \tau^{-} \bar{\nu}_{\tau}\right), \quad \mathcal{B}\left(\bar{B} \rightarrow \pi \tau^{-} \bar{\nu}_{\tau}\right)$.

We will again consider scalar and pseudoscalar NP contributions and shall follow the studies discussed in Sects. 4 and 5.

We begin by having a closer look at $\mathcal{B}\left(B^{-} \rightarrow e^{-} \bar{v}_{e}\right)$. As discussed in Sects. 1 and 3, within the SM, this branching fraction is helicity suppressed due to the tiny value of the mass of the electron. However, the presence of the pseudoscalar NP contribution $C_{P}^{\ell}$ can potentially lift the helicity suppression. In Ref. [16], we have explored an analogous mechanism that may enhance the branching fraction for the leptonic rare decays $B_{s, d} \rightarrow e^{+} e^{-}$. We now describe the main steps of our procedure using as an example the universal NP scenario: 
1. With the values of $C_{P}^{\mu}$ and $C_{P}^{\tau}$ calculated as in Sect. 5, we determine $R_{\tau}^{e}$. In the case of universal Wilson coefficients for the light leptons, we obtain

$$
R_{\tau}^{e}=\left(5.8_{-5.8}^{+8.2}\right) \times 10^{3}
$$

2. In order to obtain $\mathcal{B}\left(B^{-} \rightarrow e^{-} \bar{v}_{e}\right)$, we multiply the theoretical determination of $R_{\tau}^{e}$ with the experimental value of $\mathcal{B}\left(B^{-} \rightarrow \tau^{-} \bar{v}_{\tau}\right)$ and the relevant mass factors (see Eq. 19). We employ the experimental value in Eq. (7) which yields

$$
\mathcal{B}\left(B^{-} \rightarrow e^{-} \bar{v}_{e}\right)=\left(6.7_{-6.7}^{+9.3}\right) \times 10^{-8} .
$$

Consequently, the branching ratio for the process $B^{-} \rightarrow$ $e^{-} \bar{v}_{e}$ could be enhanced by up to four orders of magnitude with respect to the SM value given in Eq. (6). Interestingly, our determination in Eq. (81) is only one order of magnitude below the current experimental bound in Eq. (9).

For completeness, we evaluate also the observable $\mathcal{B}\left(B^{-} \rightarrow\right.$ $e^{-} \bar{v}_{e}$ ) within the four scenarios introduced in Sect. 5. The corresponding predictions are summarized in Table 2 . We illustrate graphically how our predictions for the branching fractions compare with the SM value in Fig. 18.

We proceed in an analogous way in order to determine $\mathcal{B}\left(\bar{B} \rightarrow \rho \tau^{-} \bar{\nu}_{\tau}\right)$. The steps are as follows:

1. Substitute the results for $C_{P}^{\mu}\left(\mathcal{R}_{\langle e, \mu\rangle ; \rho}^{\mu},\right)$ and $C_{P}^{\tau}\left(\mathcal{R}_{\langle e, \mu\rangle ; \rho}^{\mu}\right.$, $\left.R_{\mu}^{\tau}\right)$ obtained in Sect. 5 inside the ratio

$$
\begin{aligned}
& \mathcal{R}_{\langle e, \mu\rangle ; \rho}^{\tau ; \rho\left[m_{\tau}^{2} \leq q^{2} \leq 12\right] \mathrm{GeV}^{2}} \\
& \left.\equiv\left\langle\mathcal{B}\left(\bar{B} \rightarrow \rho \tau^{-} \bar{\nu}_{\tau}\right)\right\rangle\right|_{m_{\tau}^{2}} ^{12 \mathrm{GeV}^{2}} /\left\langle\left.\mathcal{B}\left(\bar{B} \rightarrow \rho \ell^{-} \bar{\nu}_{\ell}\right)\right|_{[\ell=e, \mu]},\right.
\end{aligned}
$$

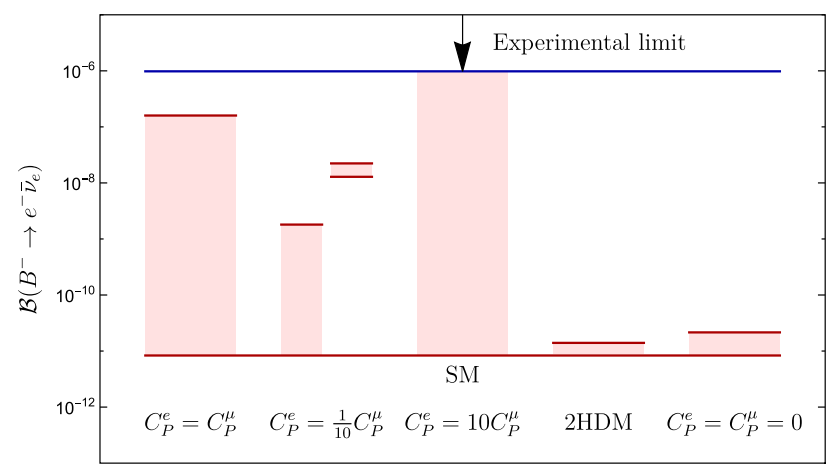

Fig. 18 Illustration of the possible enhancement of $\mathcal{B}\left(B^{-} \rightarrow e^{-} \bar{v}_{e}\right)$ for the scenarios discussed in the text. The blue line gives the current experimental upper bound on $\mathcal{B}\left(B^{-} \rightarrow e^{-} \bar{v}_{e}\right)$, whereas the red horizontal line on the bottom represents the SM value. The red regions indicate the values of the branching ratio that may be obtained constructed in analogy with $\mathcal{R}_{\langle e, \mu\rangle ; \pi}^{\langle e, \mu\rangle ; \rho\left[0 \leq q^{2} \leq 12\right] \mathrm{GeV}^{2}}$ as given by Eq. (64). In the case of universality for the light leptons our theoretical predictions are

$$
\begin{aligned}
& \mathcal{R}_{\langle e, \mu\rangle ; \rho}^{\tau ; \rho\left[m_{\tau}^{2} \leq q^{2} \leq 12\right] \mathrm{GeV}^{2}}=0.395 \pm 0.025, \\
& \mathcal{R}_{\langle e, \mu\rangle ; \rho}^{\tau ; \rho\left[m_{\tau}^{2} \leq q^{2} \leq 12\right] \mathrm{GeV}^{2}}=0.318 \pm 0.011
\end{aligned}
$$

Note that we have two solutions, corresponding to the two allowed regions in Fig. 6, which have the same $C_{P}^{\mu}$ but different values of $C_{P}^{\tau}$.

2. Multiply the theoretical determination of $\mathcal{R}_{\langle e, \mu\rangle ; \rho}^{\tau ; \rho\left[m_{\tau}^{2} \leq q^{2} \leq 12\right] \mathrm{GeV}^{2}}$ by the experimental value of the branching fraction $\left\langle\left.\mathcal{B}\left(\bar{B} \rightarrow \rho \ell^{-} \bar{v}_{\ell}\right)\right|_{[\ell=e, \mu]}\right.$. The resulting value is precisely $\left.\left\langle\mathcal{B}\left(\bar{B} \rightarrow \rho \tau^{-} \bar{\nu}_{\tau}\right)\right\rangle\right|_{m_{\tau}^{2}} ^{12 \mathrm{GeV}^{2}}$. In the universal case for light leptons, we obtain

$$
\begin{aligned}
& \left.\left\langle\mathcal{B}\left(\bar{B} \rightarrow \rho \tau^{-} \bar{\nu}_{\tau}\right)\right\rangle\right|_{m_{\tau}^{2}} ^{12 \mathrm{GeV}^{2}}=(7.81 \pm 0.66) \times 10^{-5}, \\
& \left.\left\langle\mathcal{B}\left(\bar{B} \rightarrow \rho \tau^{-} \bar{\nu}_{\tau}\right)\right\rangle\right|_{m_{\tau}^{2}} ^{12 \mathrm{GeV}^{2}}=(6.30 \pm 0.45) \times 10^{-5}
\end{aligned}
$$

We have also estimated $\left.\left\langle\mathcal{B}\left(\bar{B} \rightarrow \rho \tau^{-} \bar{v}_{\tau}\right)\right\rangle\right|_{m_{\tau}^{2}} ^{12 \mathrm{GeV}^{2}}$ for the different models introduced in Sect. 5. Our results are presented in fourth column of Table 2 and are illustrated in Fig. 19. We observe that the predictions are very stable with respect to the model under consideration. However, a measurement of this observable can be used to distinguish between the two different solutions for $C_{P}^{\tau}$. The strategies described in this section are schematically presented in Fig. 16.

In order to predict $\left\langle\mathcal{B}\left(\bar{B} \rightarrow \pi \tau^{-} \bar{\nu}_{\tau}\right)\right\rangle$, we require sensitivity on $C_{S}^{\tau}$. Unfortunately, none of the observables considered in our analysis give us direct access to this coefficient.

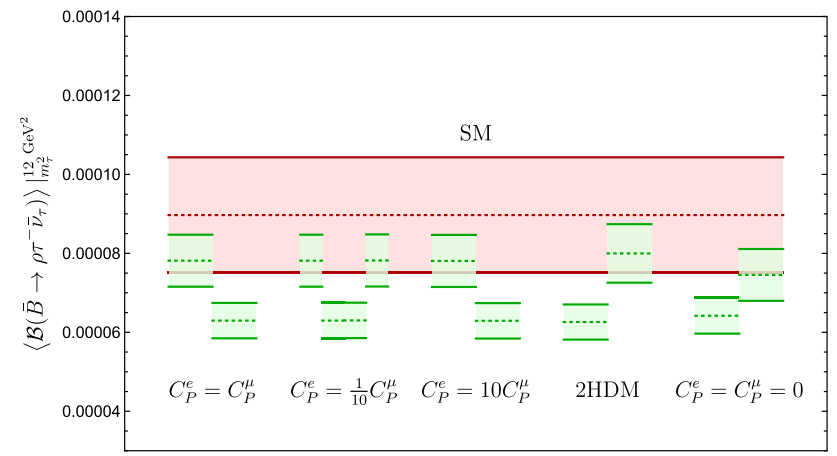

Fig. 19 Illustration of the possible values that $\mathcal{B}\left(\bar{B} \rightarrow \rho \tau^{-} \bar{\nu}_{\tau}\right)$ may take for the scenarios discussed in the text. The red horizontal band gives the SM value, whereas the green regions indicate the values of the branching ratio that may be obtained 
Therefore, in order to make predictions for this observable, we need to make extra assumptions that in general will be model dependent. For instance, the 2HDM gives us access to $C_{S}^{\tau}$ through Eq. (16), yielding

$C_{S}^{\tau}=\frac{m_{\tau}}{m_{\mu}} C_{S}^{\mu}=\frac{m_{\tau}}{m_{\mu}} C_{P}^{\mu}$.

To finally extract $\left\langle\mathcal{B}\left(\bar{B} \rightarrow \pi \tau^{-} \bar{\nu}_{\tau}\right)\right\rangle$, we consider the observable

$\mathcal{R}_{\langle e, \mu\rangle ; \pi}^{\tau ; \pi} \equiv\left\langle\mathcal{B}\left(\bar{B} \rightarrow \pi \tau^{-} \bar{v}_{\tau}\right)\right\rangle /\left\langle\left.\mathcal{B}\left(\bar{B} \rightarrow \pi \ell^{-} \bar{v}_{\ell}\right)\right|_{[\ell=e, \mu]}\right.$,

which can then be multiplied by the experimental value of $\left\langle\left.\mathcal{B}\left(\bar{B} \rightarrow \pi \ell^{-} \bar{\nu}_{\ell}\right)\right|_{[\ell=e, \mu]} \quad\right.$ to obtain $\left\langle\mathcal{B}\left(\bar{B} \rightarrow \pi \tau^{-} \bar{\nu}_{\tau}\right)\right\rangle$. Employing the relations in Eq. (85) and the experimental value in Eq. (57) we get

$$
\begin{aligned}
\left\langle\mathcal{B}\left(\bar{B} \rightarrow \pi \tau^{-} \bar{\nu}_{\tau}\right)\right\rangle & =(0.91 \pm 0.17) \times 10^{-4}, \\
\left\langle\mathcal{B}\left(\bar{B} \rightarrow \pi \tau^{-} \bar{\nu}_{\tau}\right)\right\rangle & =(1.27 \pm 0.31) \times 10^{-4} .
\end{aligned}
$$

\section{Conclusions}

We have presented a detailed analysis of leptonic $B^{-} \rightarrow$ $\ell^{-} \bar{v}_{\ell}$ decays and their semileptonic counterparts $\bar{B} \rightarrow \rho \ell^{-} \bar{v}_{\ell}$ and $\bar{B} \rightarrow \pi \ell^{-} \bar{\nu}_{\ell}$, aiming at tests of lepton flavour universality in processes caused by $b \rightarrow u \ell^{-} \bar{\nu}_{\ell}$ transitions. A key requirement to constrain the short-distance coefficients of NP operators is to consider only quantities which do not depend on $\left|V_{u b}\right|$. The point is that the values of this CKM parameter extracted from semileptonic decays assume the SM while we allow for NP contributions to these processes. Since the leptonic decays, which exhibit helicity suppression in the SM, play a key role in this endeavour, we focused on new (pseudo)-scalar operators which may lift the helicity suppression, thereby having a potentially dramatic impact on these modes.

The $B^{-} \rightarrow \ell^{-} \bar{v}_{\ell}$ decays involve actually the pseudoscalar coefficient $C_{P}^{\ell}$. Using a recent Belle result for the $B^{-} \rightarrow \mu^{-} \bar{v}_{\mu}$ branching ratio in combination with the measured $\mathcal{B}\left(B^{-} \rightarrow \tau^{-} \bar{\nu}_{\tau}\right)$, we obtained theoretically clean constraints in the $C_{P}^{\mu}-C_{P}^{\tau}$ plane. One branch of the solutions is consistent with the SM picture within the current uncertainties. Thanks to the lift of the helicity suppression, we obtain a remarkably constrained picture despite the significant experimental uncertainty for the $B^{-} \rightarrow \mu^{-} \bar{v}_{\mu}$ mode.

In order to further constrain the pseudoscalar NP coefficients, we employ the semileptonic $\bar{B} \rightarrow \rho \ell^{-} \bar{\nu}_{\ell}$ modes which involve $C_{P}^{\ell}$ as well. While the leptonic decays depend on the $B^{-}$decay constant as the only non-perturbative parameter, the semileptonic decay requires a variety of hadronic form factors which can be determined by means of QCD sum rule and lattice calculations. Using results available in the literature, we have performed a comprehensive study of the available data. Interestingly, to the best of our knowledge, measurements of differential decay rates of $\bar{B} \rightarrow \rho \ell^{-} \bar{v}_{\ell}$ for $\ell=\mu$ and $\ell=e$ are not available. It would be important for probing violations of lepton flavour universality if experimental collaborations would report such analyses. We obtain a picture which is consistent with the $\mathrm{SM}$ at the $1 \sigma$ level, taking both experimental and theoretical uncertainties into account.

The general low-energy effective Hamiltonian including NP effects has also a scalar operator which does not contribute to the $B^{-} \rightarrow \ell^{-} \bar{v}_{\ell}$ and $\bar{B} \rightarrow \rho \ell^{-} \bar{v}_{\ell}$ modes but has an impact on the semileptonic $\bar{B} \rightarrow \pi \ell^{-} \bar{\nu}_{\ell}$ channels. A comment similar to the one for the $\bar{B} \rightarrow \rho \ell^{-} \bar{\nu}_{\ell}$ modes applies also in this case, i.e. it would be very useful to have experimental results for electrons and muons in the final states. Making a simultaneous analysis of the leptonic $B^{-} \rightarrow \mu^{-} \bar{v}_{\mu}$ and semileptonic $\left\langle\bar{B} \rightarrow \pi \ell^{-} \bar{\nu}_{\ell}\right\rangle_{[\ell=e, \mu]}$ decays, we derived a constraint in the $C_{P}^{\mu}-C_{S}^{\mu}$ plane, showing one solution in agreement with the SM. Yet another constraint follows from the ratio of the differential $\left\langle\bar{B} \rightarrow \rho \ell^{-} \bar{\nu}_{\ell}\right\rangle_{[\ell=e, \mu]}$ and $\left\langle\bar{B} \rightarrow \pi \ell^{-} \bar{v}_{\ell}\right\rangle_{[\ell=e, \mu]}$ rates, which we discussed for various values of the momentum transfer $q^{2}$. Interestingly, for certain values, we obtain tension with the SM at the $1 \sigma$ level which will be interesting to monitor in the future. It would be very desirable to have more sophisticated non-perturbative analyses of the form factors available, in particular for the semileptonic $\bar{B} \rightarrow \rho$ transitions. In our study, we have also explored the impact of CP-violating phases of the NP coefficients.

Using the NP constraints, we could make corresponding predictions for decay observables which have not yet been measured. In particular, we find a potentially huge enhancement of the $B^{-} \rightarrow e^{-} \bar{v}_{e}$ branching ratio, lifting it up to the regime of the experimental upper bound. Moreover, we determined the CKM element $\left|V_{u b}\right|$, obtaining values in agreement with other analyses in the literature although having larger uncertainties.

The method which we proposed and explored for decays caused by $b \rightarrow u \ell^{-} \bar{v}_{\ell}$ quark-level processes can actually also be implemented for exclusive $\bar{B}$ decays originating from $b \rightarrow c \ell^{-} \bar{v}_{\ell}$ modes. In this case, the leptonic decay $B_{c}^{-} \rightarrow \ell^{-} \bar{v}_{\ell}$ channels are key ingredients. Unfortunately, these decays are very challenging from an experimental point of view and no measurements are currently available, despite the fact that many $B_{c}$ mesons are produced at the LHC. Hopefully, in the future, innovative ways will be found to get a handle on the leptonic $B_{c}$ modes.

It will be very interesting to apply the strategy presented in this paper in the future high-precision era of $B$ physics, thereby shedding more light on contributions of new (pseudo)-scalar operators and probing lepton flavour universality in yet another territory of the flavour physics landscape. 
Acknowledgements This research has been supported by the Netherlands Foundation for Fundamental Research of Matter (FOM) Programme 156, "Higgs as Probe and Portal", and by the National Organisation for Scientific Research (NWO). G.B. acknowledges the support through a Volkert van der Willigen grant of the University of Amsterdam.

Open Access This article is distributed under the terms of the Creative Commons Attribution 4.0 International License (http://creativecomm ons.org/licenses/by/4.0/), which permits unrestricted use, distribution, and reproduction in any medium, provided you give appropriate credit to the original author(s) and the source, provide a link to the Creative Commons license, and indicate if changes were made.

Funded by SCOAP ${ }^{3}$.

\section{A: Hadronic form factors}

In order to constrain the NP coefficients $C_{S}^{\ell}$ and $C_{P}^{\ell}$ through observables involving the semileptonic ratios $\mathcal{B}(\bar{B} \rightarrow$ $\left.\rho \ell^{-} \bar{\nu}_{\ell}\right)$ and $\mathcal{B}\left(\bar{B} \rightarrow \pi \ell^{-} \bar{\nu}_{\ell}\right)$, we need to estimate the nonperturbative contributions to the different branching fractions. Depending on the value of $q^{2}$, two approaches can be followed:

- QCD sum rules analyses, which apply to $0 \leq q^{2} \leq q_{\text {max }}^{2}$, with $q_{\text {max }}^{2}$ inside the interval $[12,16] \mathrm{GeV}^{2}$.

- Lattice QCD calculations, which provide results for $q^{2}$ close to the maximal leptonic momentum transfer. For $B \rightarrow \rho$ transitions we consider the interval

$$
q_{\text {max }}^{2} \leq q^{2} \leq\left(M_{B^{0}}-M_{\rho}\right)^{2}=20.29 \mathrm{GeV}^{2}
$$

on the other hand, for $B \rightarrow \pi$ processes we use

$$
q_{\max }^{2} \leq q^{2} \leq\left(M_{B^{0}}-M_{\pi}\right)^{2}=26.42 \mathrm{GeV}^{2}
$$

\section{A.1 Form factors for $\mathcal{B}\left(\bar{B} \rightarrow \rho \ell^{-} \bar{v}_{\ell}\right)$}

In the helicity basis, the hadronic form factors are given by [34,37]:

$$
\begin{aligned}
& H_{V, \pm}^{\rho}=\left(m_{B}+m_{\rho}\right) A_{1}\left(q^{2}\right) \mp \frac{2 M_{B}\left|\vec{p}_{\rho}\right|}{m_{B}+m_{\rho}} V\left(q^{2}\right) \\
& H_{V, 0}^{\rho}=-\frac{8 m_{B} m_{\rho}}{\sqrt{q^{2}}} A_{12}\left(q^{2}\right) \\
& H_{V, t}^{\rho}\left(q^{2}\right)=-\frac{2 M_{B}\left|\vec{p}_{\rho}\right|}{\sqrt{q^{2}}} A_{0}\left(q^{2}\right) \\
& H_{S}^{\rho}\left(q^{2}\right)=-\frac{2 M_{B}\left|\vec{p}_{\rho}\right|}{m_{b}+m_{u}} A_{0}\left(q^{2}\right)
\end{aligned}
$$

Following Ref. [37], the parametrization of the form factors for the range $0 \leq q^{2} \leq 14 \mathrm{GeV}^{2}$ obeys the generic expression
Table 3 Parameters used for the determination of the $\bar{B} \rightarrow \rho \ell^{-} \bar{v}_{\ell}$ form factors as given in Ref. [37]

\begin{tabular}{llll}
\hline & $\alpha_{0}^{k}$ & $\alpha_{1}^{k}$ & $\alpha_{2}^{k}$ \\
\hline$A_{0}$ & $0.356 \pm 0.042$ & $-0.833 \pm 0.204$ & $1.331 \pm 1.050$ \\
$A_{1}$ & $0.262 \pm 0.026$ & $0.394 \pm 0.139$ & $0.163 \pm 0.408$ \\
$A_{12}$ & $0.297 \pm 0.035$ & $0.759 \pm 0.197$ & $0.465 \pm 0.756$ \\
$V$ & $0.327 \pm 0.031$ & $-0.860 \pm 0.183$ & $1.802 \pm 0.965$ \\
\hline
\end{tabular}

Table 4 Mass terms required for the evaluation of the different $\bar{B} \rightarrow \rho$ form factors as given in Ref. [37]

\begin{tabular}{ll}
\hline$F_{i}$ & $m_{R_{i}} / \mathrm{GeV}$ \\
\hline$A_{0}$ & 5.279 \\
$V$ & 5.325 \\
$A_{1}, A_{12}$ & 5.724 \\
\hline
\end{tabular}

$F_{i}\left(q^{2}\right)=\frac{k_{b \rightarrow u}^{\rho}}{\left(1-q^{2} / m_{R, i}^{2}\right)} \sum_{k=1}^{3} \alpha_{k}^{i}\left[z_{\rho}\left(q^{2}, t_{0}\right)-z_{\rho}\left(0, t_{0}\right)\right]^{k}$,

for $F_{i}=A_{0}, A_{1}, A_{12}, V$. The required coefficients $\alpha_{k}^{i}$ can be found in Table 3 and the mass factors $m_{R, i}$ in Eq. (94) are evaluated according to the scheme presented in Table 4 . The function $z_{\rho}\left(q^{2}, t_{0}^{\rho}\right)$ is calculated using

$$
\begin{aligned}
z_{\rho}\left(q^{2}, t_{0}^{\rho}\right) & =\frac{\sqrt{\left(M_{B}+m_{\rho}\right)^{2}-q^{2}}-\sqrt{\left(M_{B}+m_{\rho}\right)^{2}-t_{0}^{\rho}}}{\sqrt{\left(M_{B}+m_{\rho}\right)^{2}-q^{2}}+\sqrt{\left(M_{B}+m_{\rho}\right)^{2}-t_{0}^{\rho}}}, \\
t_{0}^{\rho} & =\left(M_{B}+m_{\rho}\right)\left[\left(M_{B}+m_{\rho}\right)-2 \sqrt{M_{B} m_{\rho}}\right] .
\end{aligned}
$$

The parameter $k_{b \rightarrow u}^{\rho}$ in Eq. (94) accounts for the fact that the form factors $F_{i}$ above refer to $b \rightarrow u$ transitions as discussed in Ref. [37]. It is defined as

$k_{b \rightarrow u}^{\rho} \equiv \frac{f_{\rho^{0}}^{(u)}}{\bar{f}_{\rho}^{\rho^{I}}}$,

where

$f_{\rho^{0}}^{(u)}=(221.5 \pm 0.3) \times 10^{-3} \mathrm{GeV}$,

$\bar{f}_{\rho}^{\rho^{I}}=(213 \pm 5) \times 10^{-3} \mathrm{GeV}$.

For the high $q^{2}$ regime our only source is the lattice study in [33], where the interval $12.7 \mathrm{GeV}^{2} \leq q^{2} \leq 18.2 \mathrm{GeV}^{2}$ is taken into account. Unfortunately no analytical parametrization of the form factors in this region is provided. Therefore we use directly the distributions provided in [33]. These results are relatively old and potential underestimations of the 
Table 5 Parameters used for the determination of the $\bar{B} \rightarrow \pi \ell^{-} \bar{\nu}_{\ell}$ form factors as given in Ref. [32]

\begin{tabular}{lllll}
\hline & $b_{0}^{k}$ & $b_{1}^{k}$ & $b_{2}^{k}$ & $b_{3}^{k}$ \\
\hline$f_{+}$ & $0.407 \pm 0.015$ & $-0.65 \pm 0.16$ & $-0.46 \pm 0.88$ & $0.4 \pm 1.3$ \\
$f_{0}$ & $0.507 \pm 0.022$ & $-1.77 \pm 0.18$ & $1.27 \pm 0.81$ & $4.2 \pm 1.4$ \\
\hline
\end{tabular}

uncertainties are possible. However, to the best of our knowledge this is the only study available to investigate the high $q^{2}$ region. Updated lattice calculations are crucial to obtain a more precise assessment of the effects on the $B \rightarrow \rho$ channels discussed in this work.

The form factors obtained through the parametrization in Eq. (94) correspond to the transition $\bar{B}^{0} \rightarrow \rho^{+} \ell^{-} \bar{v}_{\ell}$. The expressions for $\bar{B}^{-} \rightarrow \rho^{0} \ell^{-} \bar{v}_{\ell}$ can then be calculated using isospin symmetry. Hence when evaluating the branching ratio for the process $\bar{B}^{-} \rightarrow \rho^{0} \ell^{-} \bar{\nu}_{\ell}$ the non-perturbative contributions $F_{i}$ discussed in this section should include the correction factor $1 / \sqrt{2}$.

\section{A.2 Form factors for $\mathcal{B}\left(\bar{B} \rightarrow \pi \ell^{-} \bar{\nu}_{\ell}\right)$}

The form factors for the process $\bar{B} \rightarrow \pi \ell^{-} \bar{v}_{\ell}$ in the helicity basis are $[34,38]$

$$
\begin{aligned}
H_{V, 0}^{\pi} & =\frac{2 M_{B}\left|\vec{p}_{\pi}\right|}{\sqrt{q^{2}}} f_{+}\left(q^{2}\right), \\
H_{V, t}^{\pi} & =\frac{M_{B}^{2}-m_{\pi}^{2}}{\sqrt{q^{2}}} f_{0}\left(q^{2}\right), \\
H_{S}^{\pi} & =\frac{M_{B}^{2}-m_{\pi}^{2}}{m_{b}-m_{u}} f_{0}\left(q^{2}\right) .
\end{aligned}
$$

To obtain $f_{+}\left(q^{2}\right)$ and $f_{0}\left(q^{2}\right)$ we use the Bourrely-CapriniLellouch (BCL) [39] parametrization:

$$
\begin{aligned}
f_{0}\left(q^{2}\right)= & \sum_{n=0}^{3} b_{n}^{0} z_{\pi}\left(q^{2}, t_{0}^{\pi}\right)^{n}, \\
f_{+}\left(q^{2}\right)= & \frac{1}{1-q^{2} / M_{B^{*}}^{2}} \\
& \sum_{n=0}^{3} b_{n}^{+}\left[z_{\pi}\left(q^{2}, t_{0}^{\pi}\right)^{n}-(-1)^{n-4} \frac{n}{4} z_{\pi}\left(q^{2}, t_{0}^{\pi}\right)^{4}\right] .
\end{aligned}
$$

The corresponding numerical coefficients $b_{n}^{0}$ and $b_{n}^{+}$are shown in Table 5 and were presented originally in Ref. [32]. They are the result of a fit to lattice data extrapolated to the full kinematical range in $q^{2}$.

The function $z_{\pi}\left(q^{2}, t_{0}^{\pi}\right)$ is analogous to $z_{\rho}\left(q^{2}, t_{0}^{\rho}\right)$ presented in Eq. (95) and is given by

$$
z_{\pi}\left(q^{2}, t_{0}^{\pi}\right)=\frac{\sqrt{\left(M_{B}+m_{\pi}\right)^{2}-q^{2}}-\sqrt{\left(M_{B}+m_{\pi}\right)^{2}-t_{0}^{\pi}}}{\sqrt{\left(M_{B}+m_{\pi}\right)^{2}-q^{2}}+\sqrt{\left(M_{B}+m_{\pi}\right)^{2}-t_{0}^{\pi}}},
$$

$$
t_{0}^{\pi}=\left(M_{B}+m_{\pi}\right)\left[\left(M_{B}+m_{\pi}\right)-2 \sqrt{M_{B} m_{\pi}}\right] .
$$

The form factors in Eq. (99) correspond to $\bar{B}^{0} \rightarrow$ $\pi^{+} \ell^{-} \bar{v}_{\ell}$. Those for $B^{-} \rightarrow \pi^{0} \ell^{-} \bar{v}_{\ell}$ should be estimated including an extra multiplicative factor of $1 / \sqrt{2}$.

\section{References}

1. S. Aoki et al., Eur. Phys. J. C 77, 112 (2017). arXiv:1607.00299 [hep-lat]

2. J. Charles et al., [CKMfitter Group], Eur. Phys. J. C 41, 1 (2005). https://doi.org/10.1140/epjc/s2005-02169-1. arXiv:hep-ph/0406184 (updated results and plots available at: http://ckmfitter.in2p3.fr)

3. C. Patrignani et al., [Particle Data Group], Chin. Phys. C 40, 100001 (2016) (and 2017 update)

4. A. Sibidanov et al. [Belle Collaboration], arXiv:1712.04123 [hepex]

5. N. Satoyama et al., [Belle Collaboration]. Phys. Lett. B 647, 67 (2007). arXiv:hep-ex/0611045

6. J.P. Lees et al., [BaBar Collaboration]. Phys. Rev. Lett. 109, 101802 (2012). arXiv:1205.5442 [hep-ex]

7. J.P. Lees et al., [BaBar Collaboration]. Phys. Rev. D 88, 072012 (2013). arXiv:1303.0571 [hep-ex]

8. M. Huschle et al., [Belle Collaboration]. Phys. Rev. D 92, 072014 (2015). arXiv:1507.03233 [hep-ex]

9. D. Becirevic, S. Fajfer, I. Nisandzic, A. Tayduganov, arXiv:1602.03030 [hep-ph]

10. Y. Sato et al., [Belle Collaboration]. Phys. Rev. D 94, 072007 (2016). arXiv:1607.07923 [hep-ex]

11. S. Hirose et al., [Belle Collaboration]. Phys. Rev. Lett. 118, 211801 (2017). arXiv:1612.00529 [hep-ex]

12. R. Aaij et al., [LHCb Collaboration]. Phys. Rev. Lett. 115, 111803 (2015). arXiv:1506.08614 [hep-ex]

13. W. Altmannshofer, P.S. Bhupal Dev, A. Soni, Phys. Rev. D 96, 095010 (2017). arXiv:1704.06659 [hep-ph]

14. W. Altmannshofer, C. Niehoff, P. Stangl, D.M. Straub, Eur. Phys. J. C 77, 377 (2017). arXiv:1703.09189 [hep-ph]

15. G. Hiller, I. Nisandzic, Phys. Rev. D 96, 035003 (2017). arXiv:1704.05444 [hep-ph]

16. R. Fleischer, R. Jaarsma, G. Tetlalmatzi-Xolocotzi, JHEP 1705, 156 (2017). arXiv:1703.10160 [hep-ph]

17. R. Fleischer, Phys. Rep. 370, 537 (2002). arXiv:hep-ph/0207108

18. S. Bar-Shalom, G. Eilam, M. Gronau, J.L. Rosner, Phys. Lett. B 694, 374 (2011). arXiv:1008.4354 [hep-ph]

19. J. Zupan, arXiv:1101.0134 [hep-ph]

20. J. Brod, J. Zupan, JHEP 1401, 051 (2014). https://doi.org/10.1007/ JHEP01(2014)051. arXiv:1308.5663 [hep-ph]

21. R. Fleischer, K.K. Vos, Phys. Lett. B 770, 319 (2017). arXiv:1606.06042 [hep-ph]

22. R. Fleischer, D. Galárraga Espinosa, R. Jaarsma, G. TetlalmatziXolocotzi, Eur. Phys. J. C 78, 1 (2018). arXiv:1709.04735 [hep-ph]

23. A. Khodjamirian, T. Mannel, N. Offen, Y.-M. Wang, Phys. Rev. D 83, 094031 (2011). arXiv:1103.2655 [hep-ph]

24. F.U. Bernlochner, Z. Ligeti, S. Turczyk, Phys. Rev. D 90, 094003 (2014). arXiv:1408.2516 [hep-ph]

25. A. Crivellin, S. Pokorski, Phys. Rev. Lett. 114, 011802 (2015). arXiv: 1407.1320 [hep-ph]

26. M. Tanaka, R. Watanabe, PTEP 2017(1), 013 B05 (2017). arXiv:1608.05207 [hep-ph]

27. R. Dutta, A. Bhol, Phys. Rev. D 96(3), 036012 (2017). arXiv:1611.00231 [hep-ph] 
28. M.A. Ivanov, J.G. Körner, C.T. Tran, Phys. Part. Nucl. Lett. 14, 669 (2017)

29. W.S. Hou, Phys. Rev. D 48, 2342 (1993)

30. A. Biswas, D.K. Ghosh, S.K. Patra, A. Shaw, arXiv:1801.03375 [hep-ph]

31. J.F. Kamenik, F. Mescia, Phys. Rev. D 78, 014003 (2008). arXiv:0802.3790 [hep-ph]

32. J.A. Bailey et al., [Phys. Rev. D] 92, 014024 (2015). arXiv: 1503.07839 [hep-lat]

33. K.C. Bowler et al., [UKQCD Collaboration]. JHEP 0405, 035 (2004). arXiv:hep-lat/0402023

34. Y. Sakaki, M. Tanaka, A. Tayduganov, R. Watanabe, Phys. Rev. D 88, 094012 (2013). arXiv:1309.0301 [hep-ph]
35. W. Altmannshofer, P. Ball, A. Bharucha, A.J. Buras, D.M. Straub, M. Wick, JHEP 0901, 019 (2009). arXiv:0811.1214 [hep-ph]

36. A. Sibidanov et al., [Belle Collaboration]. Phys. Rev. D 88, 032005 (2013). arXiv:1306.2781 [hep-ex]

37. A. Bharucha, D.M. Straub, R. Zwicky, JHEP 1608, 098 (2016). arXiv:1503.05534 [hep-ph]

38. S. Bhattacharya, S. Nandi, S.K. Patra, Phys. Rev. D 95, 075012 (2017). arXiv:1611.04605 [hep-ph]

39. C. Bourrely, I. Caprini, L. Lellouch, Phys. Rev. D 79, 013008 (2009). Erratum: Phys. Rev. D 82, 099902 (2010). https://doi.org/10.1103/PhysRevD.82.099902, https://doi.org/10. 1103/PhysRevD.79.013008. arXiv:0807.2722 [hep-ph] 